\title{
Stochastic action for tubes: Connecting path probabilities to measurement
}

\author{
Julian Kappler $\odot^{*}$ and Ronojoy Adhikari \\ DAMTP, Centre for Mathematical Sciences, University of Cambridge, Wilberforce Road, Cambridge CB3 OWA, United Kingdom
}

(Received 27 January 2020; revised manuscript received 12 March 2020; accepted 25 May 2020;

published 29 June 2020)

\begin{abstract}
The trajectories of diffusion processes are continuous but nondifferentiable, and each occurs with vanishing probability. This introduces a gap between theory, where path probabilities are used in many contexts, and experiment, where only events with nonzero probability are measurable. Here we bridge this gap by considering the probability of diffusive trajectories to remain within a tube of small but finite radius around a smooth path. This probability can be measured in experiment, via the rate at which trajectories exit the tube for the first time, thereby establishing a link between path probabilities and physical observables. Considering $N$-dimensional overdamped Langevin dynamics, we show that the tube probability can be obtained theoretically from the solution of the Fokker-Planck equation. Expressing the resulting exit rate as a functional of the path and ordering it as a power series in the tube radius, we identify the zeroth-order term as the Onsager-Machlup stochastic action, thereby elevating it from a mathematical construct to a physical observable. The higher-order terms reveal the form of the finite-radius contributions which account for fluctuations around the path. To demonstrate the experimental relevance of this action functional for tubes, we numerically sample trajectories of Brownian motion in a double-well potential, compute their exit rate, and show an excellent agreement with our analytical results. Our work shows that smooth tubes are surrogates for nondifferentiable diffusive trajectories and provide a direct way of comparing theoretical results on single trajectories, such as pathwise definitions of irreversibility, to measurement.
\end{abstract}

DOI: 10.1103/PhysRevResearch.2.023407

\section{INTRODUCTION}

Stochastic effects are ubiquitous in physical systems, and are widely modeled by diffusion processes [1-4]. Physical examples include the motion of individual colloidal particles [5-9], the dynamics of polymers and proteins [10-13], or the dynamics of active particles such as driven colloidal systems, cells, or bacteria [14,15]. Diffusion processes are also employed beyond the physical sciences, for example, in quantitative finance [16] or the dynamics of ecosystems [17].

A fundamental concern in stochastic dynamics is to meaningfully quantify the probability of a given trajectory. These probabilities fully characterize a given stochastic dynamics and are indispensable in applications. For example, pathwise definitions of irreversibility as ratios of probabilities of forward- and time-reversed trajectories are central to the field of stochastic thermodynamics [18,19]. As a second example, reaction pathways between states, obtained from the most probable path connecting them, are essential to the study of rare events such as chemical reactions or conformational changes in biomolecules [20-22].

For any diffusive dynamics, as, for example, the overdamped Langevin equation [2-4], which is the most widely

\section{*jk762@cam.ac.uk}

Published by the American Physical Society under the terms of the Creative Commons Attribution 4.0 International license. Further distribution of this work must maintain attribution to the author $(s)$ and the published article's title, journal citation, and DOI. used model for stochastic dynamics, the probability of any single trajectory is zero. Consequently, over the last decades, much work has been going into quantifying relative probabilities of Langevin paths [23-35]. However, because it is not possible to directly access experimentally the ratio of two vanishingly small quantities, hitherto these theoretical results could not be put to the experimental test. More generally, the fact that a given individual stochastic trajectory occurs with probability zero is the reason that no theoretical result pertaining to individual stochastic trajectories can be checked directly in experiment.

We here overcome this limitation, by shifting the focus from individual stochastic trajectories to the finite-radius tubular ensemble, composed of all stochastic trajectories that remain within a small but finite threshold distance $R$ from a smooth reference path $\varphi(t)$ [see Fig. 1 for an illustration]. The name "tubular ensemble" is motivated by the fact that this neighborhood around the reference path is a tube in spacetime. The probability to observe any of those stochastic trajectories, which is called the sojourn probability, is nonzero and can be measured directly in experiment or simulation, simply by counting which ratio of observed trajectories remains within the threshold distance from the reference path until the final observation time. Thus, considering this ensemble yields a systematic approach to regularizing and connecting to experiment the theoretical discussion of path probabilities, which are recovered as tubes shrink to zero radius. Importantly, our paper elevates stochastic actions, a widely used theoretical concept to quantify ratios of path probabilities, to physical observables. This allows the testing of theoretical results involving path probabilities directly in experiment. 


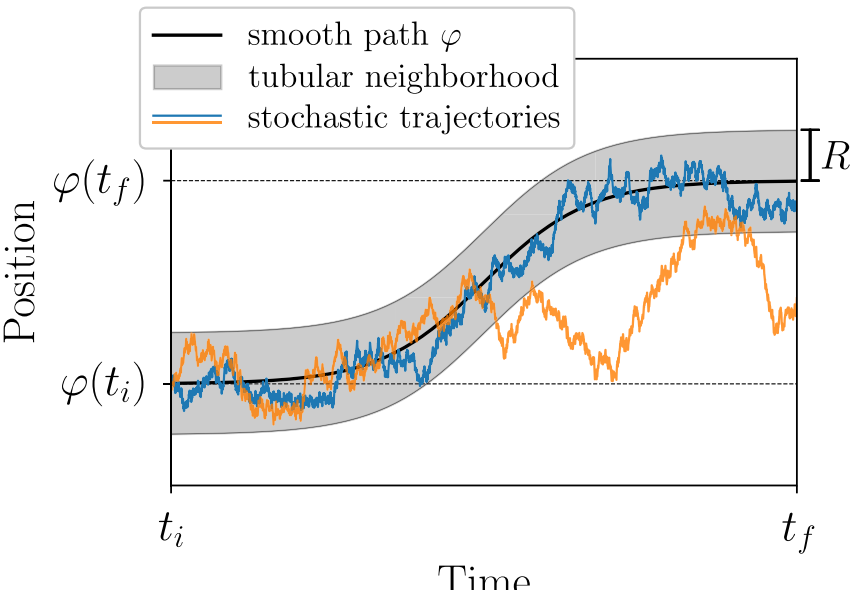

FIG. 1. For a one-dimensional system, a smooth path $\varphi$ is shown as a black solid line, around which a tube of radius $R$ is indicated as a gray shaded area. Initial and final positions of $\varphi$ are shown as horizontal dotted lines. While the blue trajectory is a realization of the Langevin Eq. (1) which stays inside the tube at all times, the orange trajectory leaves the tube before the final time $t_{f}$, and therefore contributes to the exit rate from the tube.

The relevance of the tubular ensemble, however, goes beyond serving as a bridge between theory and experiment. In physical applications, one is typically not interested in a single path, but rather in a pathway, that is a family of trajectories that remain within a threshold distance of a reference path. This is precisely the family of trajectories that the tubular ensemble describes.

Our paper establishes the tubular ensemble as a generalization of the very concept of an individual stochastic trajectory, which allows us to connect to experiment or simulation any question related to individual paths in systems subject to stochastic dynamics. For the overdamped Langevin equation, we provide a conceptually simple derivation of the sojourn probability. In the limit $R \rightarrow 0$ we recover the OnsagerMachlup (OM) stochastic action Lagrangian, which is known to characterize relative path likelihoods [23-35]; in particular, we show explicitly that this Lagrangian appears as a contribution to the exit rate with which stochastic trajectories first leave the tubular neighborhood around $\varphi$. By calculating the first radius-dependent corrections to the OM Lagrangian, we go beyond single-trajectory asymptotics.

The remainder of this paper is organized as follows. In Sec. II we discuss our general theory for $N$-dimensional Langevin dynamics. In Sec. III we illustrate our general results by considering explicitly the special case of barrier crossing in a one-dimensional system, $N=1$. We in particular show how our theoretical predictions can be compared directly to observables from simulated Langevin time series. We close in Sec. IV by summarizing our results, and discussing their further implications.

\section{THEORY}

We consider the overdamped Langevin equation, which for an $N$-dimensional coordinate $\boldsymbol{X}_{t} \equiv \boldsymbol{X}(t) \equiv$
$\left(X_{1}(t), \ldots, X_{N}(t)\right)$ is given by

$$
\dot{\boldsymbol{X}}_{t}=D \beta \boldsymbol{F}\left(\boldsymbol{X}_{t}, t\right)+\sqrt{2 D} \xi_{t},
$$

where $D=k_{\mathrm{B}} T / \gamma$ is the diffusivity; $\beta^{-1}=k_{\mathrm{B}} T$ is the inverse thermal energy with $k_{\mathrm{B}}$ the Boltzmann constant and $T$ the temperature; $\gamma$ is the friction coefficient; $\boldsymbol{F}(\boldsymbol{x}, t)$ is a deterministic, possibly time-dependent, force; and $\xi$ is Gaussian white noise with vanishing mean and unit covariance matrix. We assume that $D$ is position independent; an extension of our results to position-dependent diffusivity is discussed in the conclusions.

\section{A. The tubular ensemble}

One approach to relative path likelihoods of overdamped Langevin dynamics is to derive a formal path-integral representation of the propagator associated with Eq. (1), and then to use the resulting symbolic expression as a basis for relative path probabilities [23-28,36,37]. However, this approach suffers from ambiguities arising from the time discretization of the short-time propagator [38,39]. In essence, the formal expression one obtains depends on which of infinitely many time-discretization schemes one uses [38]; while for most purposes these discretizations are equivalent, the theoretically derived most probable path, which is sometimes thought of as representing the typical behavior of the dynamics, depends on the choice of discretization [39].

A different route towards quantifying relative path probabilities is to consider the tubular ensemble, which consists of those realizations $\boldsymbol{X}_{t}$ of the Langevin Eq. (1) that stay inside a ball of radius $R$ with its center a smooth reference path $\varphi(t)$, $t \in\left[t_{i}, t_{f}\right]$ [29-35,40], up to time $t \leqslant t_{f}$ :

$$
\mathcal{X}_{R}^{\varphi}(t) \equiv\left\{X \mid\left\|X_{s}-\varphi(s)\right\|<R \forall s \in\left[t_{i}, t\right]\right\},
$$

where $\|\boldsymbol{X}\| \equiv \sqrt{X_{1}^{2}+\ldots+X_{N}^{2}}$; see Fig. 1 for an illustration of $\mathcal{X}_{R}^{\varphi}$. We use the name "tubular ensemble" for $\mathcal{X}_{R}^{\varphi}$ because a ball with a time-dependent center is a tube in spacetime $(\boldsymbol{x}, t)$; see Fig. 1.

The corresponding sojourn probability

$$
P_{R}^{\varphi}(t) \equiv P\left(X \in \mathcal{X}_{R}^{\varphi}(t) ; X_{t_{i}} \sim P_{i}\right)
$$

is the probability for a stochastic trajectory $\boldsymbol{X}$ to remain closer than a distance $R$ to $\varphi$ until time $t$; for finite $R$ this probability of course depends on the distribution of initial positions $\boldsymbol{X}_{t_{i}} \sim$ $P_{i}$ inside the tube. Because the probability of any individual trajectory is zero for Langevin dynamics, the sojourn probability vanishes as $R \rightarrow 0$. The relative probability for two reference paths $\varphi$ and $\phi$ can still be quantified by [29-35]

$$
\frac{e^{-S[\varphi]}}{e^{-S[\phi]}} \equiv \lim _{R \rightarrow 0} \frac{P_{R}^{\varphi}\left(t_{f}\right)}{P_{R}^{\phi}\left(t_{f}\right)},
$$

where the stochastic action $S[\varphi]$, which is a functional of the smooth path $\varphi$, is found to be

$$
S[\varphi]=\int_{t_{i}}^{t_{f}} d t \mathcal{L}_{\mathrm{OM}}(\varphi(t), \dot{\varphi}(t), t),
$$

with the OM Lagrangian

$$
\mathcal{L}_{\mathrm{OM}}(\boldsymbol{\varphi}, \dot{\boldsymbol{\varphi}})=\frac{1}{4 D}[\dot{\boldsymbol{\varphi}}-D \beta \boldsymbol{F}(\boldsymbol{\varphi})]^{2}+\frac{1}{2} D \beta \nabla \cdot \boldsymbol{F}(\boldsymbol{\varphi}) .
$$


The literature concerned with deriving Eq. (6) via the ensemble Eq. (2) is rather technical [29-34], and is focused on the tubular ensemble in the singular single-trajectory limit $R \rightarrow 0$.

The key difference between the previous literature and our derivation is that, instead of working directly with the Langevin Eq. (1), we consider the equivalent description of the stochastic process inside the tube via the Fokker-Planck equation (FPE) $[3,4]$

$$
\partial_{t} P_{R}^{\varphi}(\boldsymbol{x}, t)=-\nabla \cdot\left[D \beta \boldsymbol{F}(\boldsymbol{x}, t) P_{R}^{\varphi}(\boldsymbol{x}, t)\right]+\nabla^{2}\left[D P_{R}^{\varphi}(\boldsymbol{x}, t)\right],
$$

with a time-dependent spatial domain given at time $t$ by

$$
\boldsymbol{x} \in B_{R}^{\varphi}(t) \equiv\{\boldsymbol{x} \mid\|\boldsymbol{x}-\boldsymbol{\varphi}(t)\|<R\}
$$

as illustrated by the gray shaded area in Fig. 1, and subject to absorbing boundary conditions at the tube boundary,

$$
P_{R}^{\varphi}(\boldsymbol{x}, t)=0 \quad \forall \boldsymbol{x} \in B_{R}^{\varphi}(t),
$$

so that $P_{R}^{\varphi}(\boldsymbol{x}, t)$ describes the distribution of those particles that have never left the tube until time $t$. Once Eq. (7) is solved for given initial condition $\boldsymbol{X}_{t_{i}} \sim P_{i}$, the sojourn probability up to time $t$ is simply the survival probability

$$
P_{R}^{\varphi}(t)=\int_{B_{R}^{\varphi}(t)} d^{N} \boldsymbol{x} P_{R}^{\varphi}(\boldsymbol{x}, t),
$$

where here and in the following we suppress the dependence on the initial condition $P_{i}$ unless it is relevant for the discussion. From Eq. (10) in turn we obtain the instantaneous exit rate $\alpha_{R}^{\varphi}(t)$ at which stochastic trajectories leave the tube for the first time, defined by

$$
P_{R}^{\varphi}(t)=\exp \left[-\int_{t_{i}}^{t} d s \alpha_{R}^{\varphi}(s)\right] .
$$

As we show in the following subsections, this yields

$$
\begin{aligned}
\alpha_{R}^{\varphi}(t)= & \frac{D \tilde{\lambda}_{1}^{(0)}}{R^{2}}+\mathcal{L}_{\mathrm{OM}}(\varphi(t), \dot{\varphi}(t), t) \\
& +R^{2} \mathcal{L}^{(2)}(\varphi(t), \dot{\varphi}(t), \ddot{\varphi}(t), t)+\mathcal{O}\left(R^{4}\right),
\end{aligned}
$$

where

$$
\alpha_{\text {free }} \equiv \frac{D \tilde{\lambda}_{1}^{(0)}}{R^{2}}
$$

is the free-diffusion steady-state exit rate out of a ball of radius $R$, with $\tilde{\lambda}_{1}^{(0)}$ the negative of the eigenvalue with the smallest absolute value of the Laplace operator on the unit ball $B_{1}$ with absorbing boundary conditions, $\mathcal{L}_{\mathrm{OM}}$ is the OM Lagrangian defined in Eq. (6), and $\mathcal{L}^{(2)}$ is a quadratic correction to the exit rate, which we calculate in this paper. According to Eq. (12), for small radius $R$ the exit rate is dominated by free diffusion. The OM Lagrangian is the first correction to freely diffusive exit from the tube, and with $\mathcal{L}^{(2)}$ we include finite-radius effects beyond $\mathrm{OM}$ theory. Our derivation directly relates $\mathcal{L}_{\mathrm{OM}}$ to an experimentally measurable exit rate from a fictitious tube around a smooth reference path $\varphi$; despite the appearance of the term $\alpha_{\text {free }}$ in the mathematical literature on the subject $[33,34]$, this connection between stochastic action and a physical exit rate has not been made explicit before.
In the following subsections we discuss our general theory, outlined just above, for $N$-dimensional Langevin dynamics. In Sec. II B we derive a perturbative expression for the propagator of the FPE, Eq. (7), with absorbing boundary conditions. Based on this propagator, we in Sec. II C calculate the instantaneous exit rate, defined in Eq. (11), as a power series in the tube radius $R$, which finally leads to Eq. (12).

\section{B. Perturbative solution of the FPE in the tube interior}

\section{FPE in dimensionless streaming coordinates}

To eliminate the time dependence of the spatial domain Eq. (8), we introduce the dimensionless streaming variables

$$
\tilde{t}(t) \equiv \frac{t}{\tau_{D}}, \quad \tilde{\boldsymbol{x}}(\boldsymbol{x}, t) \equiv \frac{\boldsymbol{x}-\boldsymbol{\varphi}(t)}{R},
$$

where $\tau_{D} \equiv L^{2} / D$ is the time scale on which a particle diffuses over the typical length scale $L$ of the external force $\boldsymbol{F}$. The domain for $\tilde{\boldsymbol{x}}$ is then independent of time and given by the unit ball:

$$
\tilde{\boldsymbol{x}} \in \tilde{B} \equiv\{\tilde{\boldsymbol{x}} \mid\|\tilde{\boldsymbol{x}}\|<1\} .
$$

We furthermore define a dimensionless probability density $\tilde{P}$, dimensionless force $\tilde{\boldsymbol{F}}$, and dimensionless path $\tilde{\boldsymbol{\varphi}}$ as

$$
\begin{aligned}
\tilde{P}_{\epsilon}^{\varphi}(\tilde{\boldsymbol{x}}, \tilde{t}) & \equiv R^{N} P_{R}^{\varphi}(\boldsymbol{x}, t), \\
\tilde{\boldsymbol{F}}(\tilde{\boldsymbol{x}}, \tilde{t}) & \equiv L \beta \boldsymbol{F}(\boldsymbol{x}, t), \\
\tilde{\boldsymbol{\varphi}}(\tilde{t}) & \equiv \boldsymbol{\varphi}(t) / L,
\end{aligned}
$$

where $(\boldsymbol{x}, t)$ and $(\tilde{\boldsymbol{x}}, \tilde{t})$ are related as defined in Eq. (14). Here and below, dimensionless quantities are always indicated by a tilde. In dimensionless form the FPE, Eq. (7), becomes

$$
\epsilon^{2} \partial_{\tilde{t}} \tilde{P}_{\epsilon}^{\varphi}=\tilde{\mathcal{F}}_{\text {app }} \tilde{P}_{\epsilon}^{\varphi},
$$

with the dimensionless tube radius

$$
\epsilon \equiv \frac{R}{L},
$$

and the dimensionless apparent Fokker-Planck (FP) operator $\tilde{\mathcal{F}}_{\text {app }}$, given by

$$
\tilde{\mathcal{F}}_{\text {app }} \tilde{P}_{\epsilon}^{\varphi} \equiv-\epsilon \tilde{\nabla} \cdot\left[(\tilde{\boldsymbol{F}}-\dot{\tilde{\varphi}}) \tilde{P}_{\epsilon}^{\varphi}\right]+\tilde{\nabla}^{2} \tilde{P}_{\epsilon}^{\varphi},
$$

where $\tilde{\nabla}$ denotes the gradient with respect to $\tilde{x}$ with components $\tilde{\nabla}_{j} \equiv \partial / \partial \tilde{x}_{j}$, and where $\dot{\tilde{\varphi}} \equiv \partial_{\tilde{t}} \tilde{\boldsymbol{\varphi}}$. A dot over a function in dimensionless (dimensionful) form always signifies a derivative with respect to dimensionless (dimensionful) time. For example, $\dot{\boldsymbol{\varphi}}=L / \tau_{D} \dot{\tilde{\varphi}}$. Dots are used interchangeably with the symbols $\partial_{t}$ and $\partial_{\tilde{t}}$. As can be seen directly from Eq. (21), with respect to the coordinate system $(\tilde{\boldsymbol{x}}, \tilde{t})$, the velocity of the path $\varphi$ acts as a fictitious spatially constant force inside the tube, so that we obtain an apparent total force

$$
\tilde{\boldsymbol{F}}_{\text {app }}=\tilde{\boldsymbol{F}}-\dot{\tilde{\boldsymbol{\varphi}}}
$$

which is why we call $\tilde{\mathcal{F}}_{\text {app }}$ the apparent dimensionless FP operator. In dimensionless streaming coordinates, the timedependent absorbing boundary condition, Eq. (9), becomes

$$
\tilde{P}_{\epsilon}^{\varphi}(\tilde{\boldsymbol{x}}, \tilde{t})=0 \quad \forall\|\tilde{\boldsymbol{x}}\|=1,
$$


which is independent of time. This is the principal advantage of transforming to streaming coordinates.

\section{FPE in terms of the instantaneous eigenbasis}

We expand the probability distribution $\tilde{P}_{\epsilon}^{\varphi}$ in Eq. (19) in terms of the instantaneous FP eigenstates $\tilde{\rho}_{n}(\tilde{\boldsymbol{x}}, \tilde{t})$ as

$$
\tilde{P}_{\epsilon}^{\varphi}(\tilde{\boldsymbol{x}}, \tilde{t})=\sum_{m=1}^{\infty} \tilde{a}_{m}(\tilde{t}) \tilde{\rho}_{m}(\tilde{\boldsymbol{x}}, \tilde{t}) .
$$

At time $\tilde{t}$ the eigenvalues $-\tilde{\lambda}_{n}(\tilde{t})$ and eigenfunctions $\tilde{\rho}_{n}(\tilde{\boldsymbol{x}}, \tilde{t})$ of the apparent dimensionless FP operator $\tilde{\mathcal{F}}_{\text {app }}(\tilde{t})$ fulfill the eigenvalue equation

$$
\tilde{\mathcal{F}}_{\text {app }}(\tilde{t}) \tilde{\rho}_{n}(\tilde{\boldsymbol{x}}, \tilde{t})=-\tilde{\lambda}_{n}(\tilde{t}) \tilde{\rho}_{n}(\tilde{\boldsymbol{x}}, \tilde{t})
$$

and the absorbing boundary conditions $\tilde{\rho}_{n}(\tilde{\boldsymbol{x}}, \tilde{t})=0$ for $\|\tilde{\boldsymbol{x}}\|=1$. We assume the eigenvalues to be ordered, i.e., $\tilde{\lambda}_{n} \leqslant$ $\tilde{\lambda}_{m}$ for $n<m$, and due to the absorbing boundary condition we have $\tilde{\lambda}_{1}>0$. We assume that at any time $\tilde{t}$ there exists a steady-state solution $\tilde{\rho}_{\mathrm{SS}}(\tilde{\boldsymbol{x}}, \tilde{\boldsymbol{t}})$ of Eq. (19) with reflecting boundary conditions at $\tilde{B}$; we do not require $\tilde{\rho}_{\mathrm{SS}}$ to be normalized. Using this instantaneous steady state we introduce the instantaneous inner product

$$
\langle f, g\rangle \equiv \int_{\tilde{B}} d^{N} \tilde{\boldsymbol{x}} f(\tilde{\boldsymbol{x}}) g(\tilde{\boldsymbol{x}}) / \tilde{\rho}_{\mathrm{SS}}(\tilde{\boldsymbol{x}}, \tilde{t}) .
$$

With respect to this inner product, the FP operator $\tilde{\mathcal{F}}_{\text {app }}$ is self-adjoint so that the absorbing-boundary eigenfunctions $\tilde{\rho}_{n}$ can be chosen orthogonal at each time $\tilde{t}$ [4]. If at any time $\tilde{t}$ the force $\boldsymbol{F}(\boldsymbol{x}, t)$ inside the domain $B_{R}^{\varphi}(t)$ originates from a potential $U(\boldsymbol{x}, t)$, such that $\boldsymbol{F}=-\nabla U$, then the instantaneous steady-state solution is given by

$$
\tilde{\rho}_{\mathrm{SS}}(\tilde{\boldsymbol{x}}, \tilde{t})=\exp [-\epsilon \tilde{U}(\tilde{\boldsymbol{x}}, \tilde{t})-\epsilon \tilde{\boldsymbol{x}} \cdot \dot{\tilde{\boldsymbol{\varphi}}}],
$$

where $\tilde{U}(\tilde{\boldsymbol{x}}, \tilde{t}) \equiv \beta U(x, t)$, and the dot indicates the standard Euclidean inner product on $\mathbb{R}^{N}$. We emphasize that Eq. (27) does not require a global potential for $\boldsymbol{F}$, but only a local potential inside the ball $B_{R}^{\varphi}(t)$. If such a local potential does not exist, the instantaneous nonequilibrium steady state $\tilde{\rho}_{\mathrm{SS}}$ has to be determined by other means [41].

Expanding the probability distribution $\tilde{P}_{\epsilon}^{\varphi}$ in Eq. (19) in terms of the instantaneous FP eigenstates as given by Eq. (24), and projecting the equation onto $\tilde{\rho}_{n}$ using the inner product Eq. (26), yields

$$
-\dot{\tilde{a}}_{n}=\frac{\tilde{\lambda}_{n}}{\epsilon^{2}} \tilde{a}_{n}+\sum_{m=1}^{\infty} \frac{\left\langle\tilde{\rho}_{n}, \dot{\tilde{\rho}}_{m}\right\rangle}{\left\langle\tilde{\rho}_{n}, \tilde{\rho}_{n}\right\rangle} \tilde{a}_{m},
$$

where $n \in \mathbb{N}$ and a dot here denotes a derivative with respect to $\tilde{t}$. Because the apparent FP operator is time dependent, both the eigenvalues $\tilde{\lambda}_{n}$ and the inner products $\left\langle\tilde{\rho}_{n}, \dot{\tilde{\rho}}_{m}\right\rangle$ and $\left\langle\tilde{\rho}_{n}, \tilde{\rho}_{n}\right\rangle$ are functions of $\tilde{t}$. The FPE, Eq. (7), with absorbing boundary conditions is equivalent to Eq. (28); once the latter is solved, the dimensionless probability density inside the tube is obtained from Eq. (24), which can be recast in physical units using Eq. (16).

Since $\tilde{\mathcal{F}}_{\text {app }}$ depends on $\epsilon$, so do the quantities $\tilde{\lambda}_{n},\left\langle\tilde{\rho}_{n}, \dot{\tilde{\rho}}_{m}\right\rangle$, and $\left\langle\tilde{\rho}_{n}, \tilde{\rho}_{n}\right\rangle$, which appear in Eq. (28). From Eq. (21) it is apparent that the ratio of the drift to the diffusion is of order $\epsilon$ and, therefore, to lowest order the spectrum is that of a free diffusion inside a unit ball. The eigenvalues, eigenfunctions, and steady-state distributions are independent of $\tilde{t}$ at this order, and therefore any time dependence of the eigenfunctions must be at least of order $\epsilon$. This implies that the ratio of the off-diagonal to diagonal terms in Eq. (28) is at least of order $\epsilon^{3}$. Thus, mode-coupling effects are subdominant and the uncoupled dynamics provides a good first approximation for small $\epsilon$. In the context of time-dependent perturbation theory in quantum mechanics, this is known as the adiabatic approximation [42].

\section{Perturbative calculation of the instantaneous FP spectrum}

In Appendix A, we discuss in detail the calculation of both the instantaneous eigenvalues and eigenfunctions as perturbation series in $\epsilon$,

$$
\tilde{\lambda}_{n}=\sum_{k=0}^{\infty} \epsilon^{k} \tilde{\lambda}_{n}^{(k)}, \quad \tilde{\rho}_{n}=\sum_{k=0}^{\infty} \epsilon^{k} \tilde{\rho}_{n}^{(k)},
$$

and calculate explicit expressions for the eigenvalues $\tilde{\lambda}_{n}$ to order $\epsilon^{3}$, and for the eigenfunctions $\tilde{\rho}_{n}$ to order $\epsilon$. For $n=1$, and if the force $\boldsymbol{F}$ inside the tube is given by a potential also for $n>1$, we furthermore calculate explicitly the contribution $\tilde{\rho}_{n}^{(2)}$ at order $\epsilon^{2}$.

\section{Perturbative solution of the FPE}

In Appendix B we in detail derive a solution to Eq. (28), given by

$$
\begin{aligned}
\tilde{a}_{1}(\tilde{t}) \approx & \exp \left[-\frac{1}{\epsilon^{2}} \int_{\tilde{t}_{i}}^{\tilde{t}} d \tilde{t}^{\prime} \tilde{\Lambda}_{1}\left(\tilde{t}^{\prime}\right)\right] \\
\times & {\left[\tilde{a}_{1}\left(\tilde{t}_{i}\right)-\left.\epsilon^{2} \sum_{m=2}^{\infty} \frac{\left\langle\tilde{\rho}_{1}, \dot{\tilde{\rho}}_{m}\right\rangle}{\left\langle\tilde{\rho}_{1}, \tilde{\rho}_{1}\right\rangle}\right|_{\tilde{t}_{i}} \frac{\tilde{a}_{m}\left(\tilde{t}_{i}\right)}{\Delta \tilde{\Lambda}_{m 1}\left(\tilde{t}_{i}\right)}+\mathcal{O}\left(\epsilon^{k}\right)\right], } \\
& \tilde{a}_{n}(\tilde{t}) \approx-\left.\epsilon^{2} \frac{\left\langle\tilde{\rho}_{n}, \dot{\tilde{\rho}}_{1}\right\rangle}{\left\langle\tilde{\rho}_{n}, \tilde{\rho}_{n}\right\rangle}\right|_{\tilde{t}} \frac{\tilde{a}_{1}(\tilde{t})}{\Delta \tilde{\Lambda}_{n 1}(\tilde{t})}+\mathcal{O}\left(\epsilon^{k}\right),
\end{aligned}
$$

where $n>1$ in Eq. (31), for a one-dimensional system $N=1$ we have $k=6$ and for $N \geqslant 2$ we have $k=5$, and we define

$$
\begin{gathered}
\tilde{\Lambda}_{n} \equiv \tilde{\lambda}_{n}+\epsilon^{2} \frac{\left\langle\tilde{\rho}_{n}, \dot{\tilde{\rho}}_{n}\right\rangle}{\left\langle\tilde{\rho}_{n}, \tilde{\rho}_{n}\right\rangle}, \\
\Delta \tilde{\Lambda}_{m n} \equiv \tilde{\Lambda}_{m}-\tilde{\Lambda}_{n} .
\end{gathered}
$$

The solution Eqs. (30) and (31) is valid after an initial transient time, i.e., for

$$
\tilde{t}-\tilde{t}_{i} \gtrsim \tilde{\tau}_{\text {rel }} \equiv \frac{\epsilon^{2}}{\Delta \Lambda_{21}},
$$

and neglects terms that are exponentially small as compared to Eqs. (30) and (31).

The form of Eqs. (30) and (31) allows for an intuitive interpretation. Initially all eigenmodes are excited, with their respective amplitude $\tilde{a}_{n}\left(\tilde{t}_{i}\right)$ determined by the initial condition. The dynamics of each mode is dominated by the adiabatic exponential decay, and after an initial relaxation time the mode $n=1$ (which decays slowest) dominates the probability distribution Eq. (24); this is represented by the first term in 
the bracket in Eq. (30). The leading-order effect of the mode coupling is twofold. First, during their initial decay the modes $n>1$ can transfer some of their initial amplitude $\tilde{a}_{n}\left(\tilde{t}_{i}\right)$ to the $n=1$ mode, as described by the second term in the bracket in Eq. (30). Second, after their initial decay the $n>1$ modes can be excited instantaneously by the lowest mode $n=1$, as described by Eq. (31).

For a particle initially localized at $\tilde{x}_{i}$, we have a delta-peak initial condition, $\tilde{P}_{\epsilon}^{\varphi}\left(\tilde{\boldsymbol{x}}, \tilde{t}_{i}\right) \equiv \tilde{P}_{i}(\tilde{\boldsymbol{x}})=\delta\left(\tilde{\boldsymbol{x}}-\tilde{\boldsymbol{x}}_{i}\right)$, so that the initial amplitude of the $n$th mode is given by

$$
\tilde{a}_{n}\left(\tilde{t}_{i}\right)=\left.\frac{\left\langle\tilde{P}_{\epsilon}^{\varphi}, \tilde{\rho}_{n}\right\rangle}{\left\langle\tilde{\rho}_{n}, \tilde{\rho}_{n}\right\rangle}\right|_{\tilde{t}_{i}}=\frac{\tilde{\rho}_{n}\left(\tilde{\boldsymbol{x}}_{i}, \tilde{t}_{i}\right)}{\left.\tilde{\rho}_{\mathrm{SS}}\left(\tilde{\boldsymbol{x}}_{i}, \tilde{t}_{i}\right)\left\langle\tilde{\rho}_{n}, \tilde{\rho}_{n}\right\rangle\right|_{\tilde{t}_{i}}} .
$$

Substituting the resulting coefficients Eq. (30) and (31) into the eigenmode expansion Eq. (24) of the propagator then yields

$$
\begin{aligned}
\tilde{P}_{\epsilon}^{\varphi}\left(\tilde{\boldsymbol{x}}, \tilde{t} \mid \tilde{\boldsymbol{x}}_{i}, \tilde{t}_{i}\right) \\
=\exp \left[-\frac{1}{\epsilon^{2}} \int_{\tilde{t}_{i}}^{\tilde{t}} d \tilde{t}^{\prime} \tilde{\Lambda}_{1}\left(\tilde{t}^{\prime}\right)\right] \frac{1}{\left.\tilde{\rho}_{\mathrm{SS}}\left(\tilde{\boldsymbol{x}}_{i}, \tilde{t}_{i}\right)\left\langle\tilde{\rho}_{1}, \tilde{\rho}_{1}\right\rangle\right|_{\tilde{t}_{i}}} \\
\times\left[\tilde{\rho}_{1}(\tilde{\boldsymbol{x}}, \tilde{t})-\left.\epsilon^{2} \sum_{m=2}^{\infty} \frac{1}{\Delta \tilde{\Lambda}_{m 1}(\tilde{t})} \frac{\left\langle\tilde{\rho}_{m}, \dot{\tilde{\rho}}_{1}\right\rangle}{\left\langle\tilde{\rho}_{m}, \tilde{\rho}_{m}\right\rangle}\right|_{\tilde{t}} \tilde{\rho}_{m}(\tilde{\boldsymbol{x}}, \tilde{t})\right] \\
\times\left[\tilde{\rho}_{1}\left(\tilde{\boldsymbol{x}}_{i}, \tilde{t}_{i}\right)-\left.\epsilon^{2} \sum_{m=2}^{\infty} \frac{1}{\Delta \tilde{\Lambda}_{m 1}\left(\tilde{t}_{i}\right)} \frac{\left\langle\tilde{\rho}_{1}, \dot{\tilde{\rho}}_{m}\right\rangle}{\left\langle\tilde{\rho}_{m}, \tilde{\rho}_{m}\right\rangle}\right|_{\tilde{t}_{i}} \tilde{\rho}_{m}\left(\tilde{\boldsymbol{x}}_{i}, \tilde{t}_{i}\right)\right] \\
+\mathcal{O}\left(\epsilon^{k}\right),
\end{aligned}
$$

where $k=6$ for a one-dimensional system, $N=1$, and $k=5$ for $N \geqslant 2$. Equation (36) is an approximate solution to the FPE, Eq. (19), valid after an initial decay time $\tilde{\tau}_{\text {rel }}$ defined in Eq. (34). With the definitions Eqs. (32) and (33), the propagator Eq. (36) is fully expressed in terms of the instantaneous eigenvalues and eigenvectors of the FP operator. Note that Eq. (36) is factorized into a part that only depends on $(\tilde{\boldsymbol{x}}, \tilde{t})$, and a part that only depends on $\left(\tilde{\boldsymbol{x}}_{i}, \tilde{t}_{i}\right)$. Thus, while the total probability to have remained inside the tube until time $\tilde{t}$ is affected by the initial condition, after the initial relaxation time $\tilde{\tau}_{\text {rel }}$ the spatial probability distribution inside the tube is independent of the initial condition.

Using Eq. (36), we can express the solution for an arbitrary initial distribution $\tilde{P}_{i}$ inside the tube as

$$
\tilde{P}_{\epsilon}^{\varphi}\left(\tilde{\boldsymbol{x}}, \tilde{t} \mid \tilde{\boldsymbol{X}}_{\tilde{t}_{i}} \sim \tilde{P}_{i}\right)=\int_{\tilde{B}} d^{N} \tilde{\boldsymbol{x}}_{i} \tilde{P}_{\epsilon}^{\varphi}\left(\tilde{\boldsymbol{x}}, \tilde{t} \mid \tilde{\boldsymbol{x}}_{i}, \tilde{t}_{i}\right) \tilde{P}_{i}\left(\tilde{\boldsymbol{x}}_{i}\right),
$$

from which the survival probability, Eq. (10), follows in dimensionless form as

$$
\tilde{P}_{\epsilon}^{\varphi}\left(\tilde{t} \mid \tilde{\boldsymbol{X}}_{\tilde{t}_{i}} \sim \tilde{P}_{i}\right)=\int_{\tilde{B}} d^{N} \tilde{\boldsymbol{x}} \tilde{P}_{\epsilon}^{\varphi}\left(\tilde{\boldsymbol{x}}, \tilde{t} \mid \tilde{\boldsymbol{X}}_{\tilde{t}_{i}} \sim \tilde{P}_{i}\right) .
$$

Complementary to the survival probability is the normalized probability density $\tilde{P}_{\epsilon}^{n, \varphi}$ inside the tube at any time $\tilde{t}$, defined as

$$
\tilde{P}_{\epsilon}^{n, \varphi}(\tilde{\boldsymbol{x}}, \tilde{t}) \equiv \frac{\tilde{P}_{\epsilon}^{\varphi}\left(\tilde{\boldsymbol{x}}, \tilde{t} \mid \tilde{\boldsymbol{X}}_{\tilde{t}_{i}} \sim \tilde{P}_{i}\right)}{\int_{\tilde{B}} d^{N} \tilde{\boldsymbol{x}}^{\prime} \tilde{\boldsymbol{P}}_{\epsilon}^{\varphi}\left(\tilde{\boldsymbol{x}}^{\prime}, \tilde{t} \mid \tilde{\boldsymbol{X}}_{\tilde{t}_{i}} \sim \tilde{P}_{i}\right)},
$$

which describes the distribution inside the tube of those particles that have stayed until the current time $\tilde{t}$. Using
Eqs. (36) and (37), the distribution Eq. (39) can be shown to be independent of $\tilde{P}_{i}$.

\section{Exit rate from the tube}

For a particle starting at time $t_{i}$ according to a distribution $\boldsymbol{X}_{t_{i}} \sim P_{i}$ inside the tube, the instantaneous exit rate is given by

$$
\alpha_{R}^{\varphi}(t)=-\frac{\dot{P}_{R}^{\varphi}(t)}{P_{R}^{\varphi}(t)},
$$

where $P_{R}^{\varphi}(t) \equiv P_{R}^{\varphi}\left(t \mid \boldsymbol{X}_{t_{i}} \sim P_{i}\right)$ is the survival probability defined in Eq. (10). Using Eqs. (14) and (16), the dimensionless instantaneous exit rate Eq. (40) is defined as

$$
\tilde{\alpha}_{\epsilon}^{\varphi}(\tilde{t}) \equiv \tau_{D} \alpha_{R}^{\varphi}(t)=-\frac{\dot{\tilde{P}}_{\epsilon}^{\varphi}(\tilde{t})}{\tilde{P}_{\epsilon}^{\varphi}(\tilde{t})}
$$

where the dot denotes a derivative with respect to $\tilde{t}$, and $\tilde{P}_{\epsilon}^{\varphi}(\tilde{t}) \equiv \tilde{P}_{\epsilon}^{\varphi}\left(\tilde{t} \mid \tilde{X}_{\tilde{t}_{i}} \sim \tilde{P}_{i}\right)$ is the survival probability in dimensionless form, with $\tilde{P}_{i}(\tilde{\boldsymbol{x}})=R^{N} P_{i}(\boldsymbol{x})$. Using the steadystate FP solution Eqs. (36)-(38), the exit rate Eq. (41) is evaluated to yield

$$
\tilde{\alpha}_{\epsilon}^{\varphi}(\tilde{t})=\frac{\tilde{\lambda}_{1}}{\epsilon^{2}}+\frac{\left\langle\tilde{\rho}_{1}, \dot{\tilde{\rho}}_{1}\right\rangle}{\left\langle\tilde{\rho}_{1}, \tilde{\rho}_{1}\right\rangle}-\frac{\dot{\tilde{\mathcal{I}}}_{1}}{\tilde{\mathcal{I}}_{1}}+\mathcal{O}\left(\epsilon^{4}\right),
$$

with

$$
\tilde{\mathcal{I}}_{n}(\tilde{t}) \equiv \int_{\tilde{B}} d^{N} \tilde{\boldsymbol{x}} \tilde{\rho}_{n}(\tilde{\boldsymbol{x}}, \tilde{t}),
$$

and where we used that $\left\langle\tilde{\rho}_{m}, \dot{\tilde{\rho}}_{1}\right\rangle \tilde{\mathcal{I}}_{m}$ is of order $\epsilon^{2}$ (see Appendix A3).

Equation (42), which is valid after the initial transient decay time $\tilde{\tau}_{\text {rel }}$ defined in Eq. (34), is independent of the initial distribution $\tilde{P}_{i}$; this is because in Eq. (36) the initial condition only contributes an overall prefactor independent of $(\tilde{\boldsymbol{x}}, \tilde{t})$, which does not affect the relative change of particles inside the tube quantified by Eq. (41). With Eq. (42) the instantaneous exit rate is expressed solely in terms of the instantaneous FP spectrum inside the tube. Expanding the quantities that appear in Eq. (42) in powers of $\epsilon$, and using the symmetry properties of these quantities (see Appendix A), a power-series expansion of the exit rate is obtained as

$$
\tilde{\alpha}_{\epsilon}^{\varphi}=\frac{\tilde{\lambda}_{1}^{(0)}}{\epsilon^{2}}+\tilde{\alpha}^{(0)}+\epsilon^{2} \tilde{\alpha}^{(2)}+\mathcal{O}\left(\epsilon^{4}\right),
$$

where

$$
\begin{gathered}
\tilde{\alpha}_{\text {free }}=\frac{\tilde{\lambda}_{1}^{(0)}}{\epsilon^{2}}, \\
\tilde{\alpha}^{(0)}=\tilde{\lambda}_{1}^{(2)}=\tau_{D} \mathcal{L}_{\mathrm{OM}}, \\
\tilde{\alpha}^{(2)}=\tilde{\lambda}_{1}^{(4)}+\frac{\left\langle\tilde{\rho}_{1}, \dot{\tilde{\rho}}_{1}\right\rangle^{(2)}}{\left\langle\tilde{\rho}_{1}, \tilde{\rho}_{1}\right\rangle^{(0)}}-\frac{\dot{\tilde{\mathcal{I}}}_{1}^{(2)}}{\tilde{\mathcal{I}}_{1}^{(0)}},
\end{gathered}
$$

where at Eq. (46) we use the perturbative result for $\tilde{\lambda}_{n}^{(2)}$ (see Appendix A), the definition of the OM Lagrangian $\mathcal{L}_{\mathrm{OM}}$ is given in Eq. (6), and

$$
\tilde{\mathcal{I}}_{n}^{(k)}(\tilde{t}) \equiv \int_{\tilde{B}} d^{N} \tilde{\boldsymbol{x}} \tilde{\rho}_{n}^{(k)}(\tilde{\boldsymbol{x}}, \tilde{t}) .
$$


Note that we suppress the dependence on $\varphi$ in the notation of the $\tilde{\alpha}^{(k)}$, and that $\tilde{\alpha}_{\text {free }}$ is independent of $\varphi$.

Using Eq. (41) the exit rate in physical units can be obtained from Eqs. (44)-(47); note that according to Eq. (41) a scaling $\epsilon^{k}$ in $\tilde{\alpha}_{\epsilon}^{\varphi}$ (dimensionless form) translates to a scaling $R^{k}$ in $\alpha_{R}^{\varphi}$ (physical units). The order- $\epsilon^{2}$ term in Eq. (12) is thus given by $\mathcal{L}^{(2)}=\tilde{\alpha}^{(2)} /\left(\tau_{D} L^{2}\right)$; according to Eq. (21) the instantaneous FP spectrum depends on $(\varphi, \dot{\varphi})$; because of the additional time derivative in Eq. (47), the term $\mathcal{L}^{(2)}$ additionally depends on $\ddot{\varphi}$.

Equations (44)-(47), which express the exit rate $\tilde{\alpha}_{\epsilon}^{\varphi}$ fully in terms of the perturbative spectrum of the FP operator inside the tube, are one of the main results of this paper. The equations show that for small tube radius $\epsilon \ll 1$ the exit from the tube is dominated by the steady-state free-diffusion exit rate given by Eq. (45); this is consistent with the fact that the Langevin Eq. (1) is on short times dominated by the noise term $\boldsymbol{\xi}$ (as opposed to the deterministic force $\boldsymbol{F}$ ). From Eq. (44) we see that the free-diffusion exit rate in fact diverges as $1 / \epsilon^{2}$, which gives a physical picture as to why the probability for observing the single path $\varphi$ is zero.

According to Eqs. (44) and (46), the first correction to the free-diffusion exit rate, which occurs at order $\epsilon^{0}$, is given by the OM Lagrangian $\mathcal{L}_{\mathrm{OM}}$; this establishes a direct link between $\mathcal{L}_{\mathrm{OM}}$ and the physical observable $\alpha_{R}^{\varphi}$. The next correction Eq. (47), which is quadratic in the tube radius, is still in the adiabatic limit, meaning that only the $n=1$ eigenvalue and eigenfunction appear in Eq. (47).

\section{ONE-DIMENSIONAL SYSTEMS AND NUMERICAL EXPERIMENTS}

In the present section we consider the special case of a onedimensional system, $N=1$, for which it is straightforward to calculate explicit expressions for the results derived in Sec. II. To illustrate and verify our perturbative analytical results, we compare to numerical simulations throughout; in Sec. III A we introduce the corresponding example system, a double-well system with a barrier-crossing transition path $\varphi$. While in Sec. III B we discuss the normalized probability density inside the tube, we in Sec. III C consider the exit rate.

\section{A. Model}

For a length scale $L$ and a time scale $T$, we consider the Langevin Eq. (1) with a diffusion coefficient $D=L^{2} / T$, so that $\tau_{D}=T$. We consider a force $F$ that is given as the gradient of a potential, $F(x)=-\left(\partial_{x} U\right)(x)$, and for the potential $U(x)$ use a quartic double well,

$$
U(x)=U_{0}\left[\left(\frac{x}{L}\right)^{2}-1\right]^{2},
$$

with $\beta U_{0}=2$, as illustrated on the right-hand side of Fig. 2 . For the smooth reference path $\varphi$ we choose a barrier crossing path, parametrized as

$$
\varphi(t)=\frac{L}{\arctan (\kappa / 2)} \arctan \left[\kappa \cdot\left(\frac{t-t_{f} / 2}{\tau_{D}}\right)\right],
$$

where for $\kappa$, which controls the maximal barrier crossing speed, we use $\kappa=10$; we furthermore choose $t_{i}=0, t_{f}=\tau_{D}$.

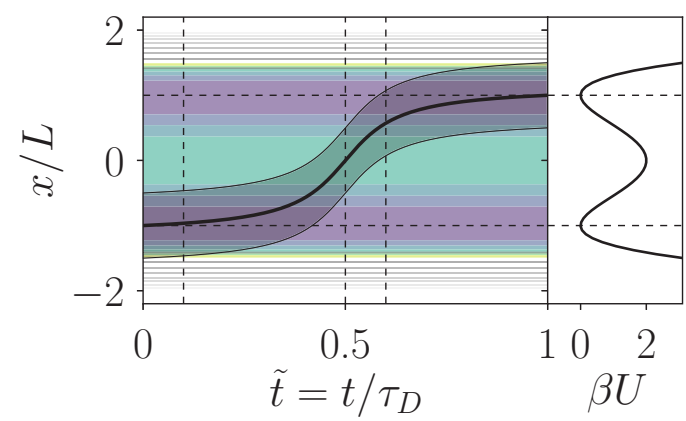

FIG. 2. Potential and path considered in the numerical examples in Sec. III. The plot on the right-hand side shows the quartic doublewell potential Eq. (49) for barrier height $\beta U_{0}=2$. In the plot on the left-hand side, the potential is shown as a color map in the background, with the two minima of the potential represented by horizontal dashed lines. The reference path $\varphi$, defined in Eq. (50), is shown as a solid black line. Around the reference path, a tube of radius $\epsilon=R / L=0.5$ is depicted by a shaded gray region. The vertical dashed lines denote the times $\tilde{t}=0.1,0.5$, and 0.6 , which are considered in Fig. 3.

The prefactor in Eq. (50) ensures that the path starts at $x=-L$ and ends at $x=L$. The reference path Eq. (50) is illustrated in Fig. 2.

\section{B. Perturbative solution of the FPE in the tube interior}

As we show in detail in Appendix C 1 , for $N=1$ the eigenvalue/eigenfunction Eq. (25) can be solved recursively for increasing $k$, and the solution at order $k$ is of the form

$$
\begin{aligned}
\tilde{\rho}_{n}^{(k)}(\tilde{x}, \tilde{t})= & \tilde{Q}_{n, \mathrm{~s}}^{(k)}(\tilde{x}, \tilde{t}) \sin \left[n \frac{\pi}{2}(\tilde{x}+1)\right] \\
& +\tilde{Q}_{n, \mathrm{c}}^{(k)}(\tilde{x}, \tilde{t}) \cos \left[n \frac{\pi}{2}(\tilde{x}+1)\right],
\end{aligned}
$$

where $\tilde{Q}_{n, \mathrm{~s}}^{(k)}(\tilde{x}, \tilde{t})$ and $\tilde{Q}_{n, \mathrm{c}}^{(k)}(\tilde{x}, \tilde{t})$ are polynomials in $\tilde{x}$ of order $\leqslant k$, and depend on $\tilde{t}$ via $\tilde{E}_{l}(\tilde{t}), 1 \leqslant l \leqslant k$, which are given by

$$
\tilde{E}_{k}(\tilde{t}) \equiv-\left.\frac{L^{k} \beta}{k !} \frac{\partial^{k-1} F}{\partial x^{k-1}}\right|_{(\varphi(t), t)}+\delta_{k, 1} \dot{\tilde{\varphi}}(\tilde{t})
$$

In Appendix C 1, we give explicit expressions for $\tilde{\lambda}_{n}^{(k)}, \tilde{Q}_{n, \mathrm{~s}}^{(k)}$, and $\tilde{Q}_{n, \mathrm{c}}^{(k)}$, up to $k=5$.

Using the perturbative spectrum Eq. (51), the propagator Eq. (36) can be calculated as a power series in $\epsilon$. From the propagator, in turn, the perturbation series for the normalized probability density $\tilde{P}_{\epsilon}^{n, \varphi}$ inside the tube is obtained using Eq. (39). It is found that $\tilde{P}_{\epsilon}^{n, \varphi}$ is of the form

$$
\begin{aligned}
\tilde{P}_{\epsilon}^{n, \varphi}(\tilde{x}, \tilde{t})= & \sum_{k=0}^{5} \epsilon^{k}\left\{\tilde{N}_{\mathrm{s}}^{(k)}(\tilde{x}, \tilde{t}) \sin \left[\frac{\pi}{2}(\tilde{x}+1)\right]\right. \\
& \left.+\tilde{N}_{\mathrm{c}}^{(k)}(\tilde{x}, \tilde{t}) \cos \left[\frac{\pi}{2}(\tilde{x}+1)\right]\right\}+\mathcal{O}\left(\epsilon^{6}\right),
\end{aligned}
$$

where the coefficients $\tilde{N}_{\mathrm{s}}^{(k)}(\tilde{x}, \tilde{t})$ and $\tilde{N}_{\mathrm{c}}^{(k)}(\tilde{x}, \tilde{t})$ are polynomials in $\tilde{x}$ of order $\leqslant k$, and depend on $\tilde{t}$ via $\tilde{E}_{l}(\tilde{t}), 1 \leqslant l \leqslant k$, as defined in Eq. (52). The explicit expressions for $\tilde{N}_{\mathrm{s}}^{(k)}$ and $\tilde{N}_{\mathrm{c}}^{(k)}$ for $k \leqslant 5$ are given in Appendix C 3 . 

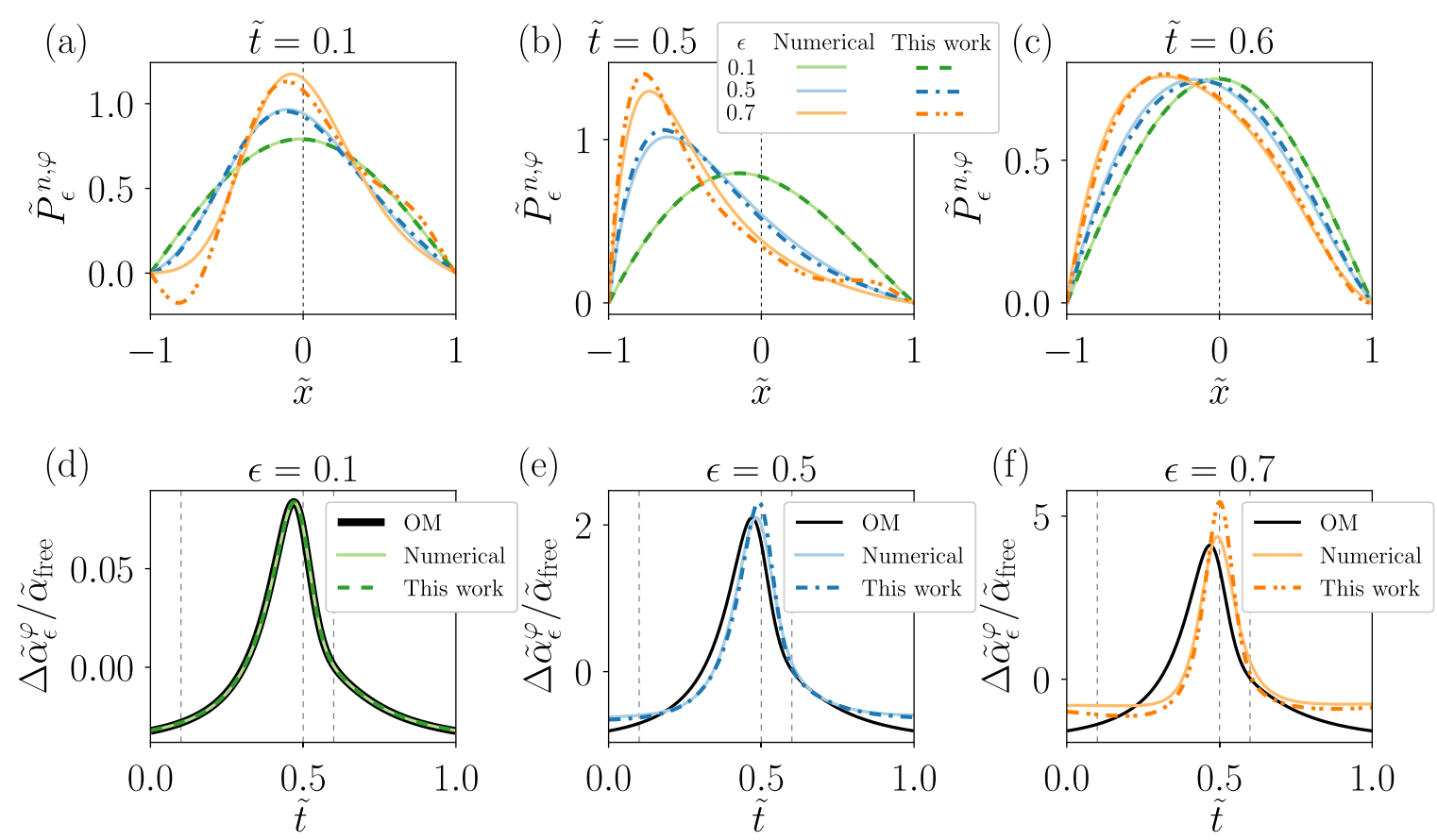

FIG. 3. Subplots (a), (b), and (c) show the normalized probability density $\tilde{P}_{\epsilon}^{n, \varphi}(\tilde{x}, \tilde{t})$, defined in Eq. (53), as a function of position $\tilde{x}$ for time (a) $\tilde{t}=0.1$, (b) $\tilde{t}=0.5$, and (c) $\tilde{t}=0.6$, and for tube radius $\epsilon=0.1$ (green), $\epsilon=0.3$ (blue), and $\epsilon=0.5$ (orange). The legend given in subplot (b) is valid for subplots (a), (b), and (c). Solid colored lines denote results from numerical simulation of the FPE, Eq. (19) (see Appendix C 5 for details on the numerical algorithm). Colored broken lines denote the perturbative result Eq. (53), calculated to order $\epsilon^{5}$. Vertical dashed lines indicate the tube center $\tilde{x}=0$. Subplots (d), (e), and (f) show the exit rate $\tilde{\alpha}_{\epsilon}^{\varphi}$, defined in Eq. (44), as a function of time $\tilde{t}$, for tube radii (d) $\epsilon=0.1$, (e) $\epsilon=0.5$, and (f) $\epsilon=0.7$. From all rates the free-diffusion exit rate is subtracted and the result is divided by the free-diffusion exit rate, as defined in Eq. (57). Colored solid lines denote exit rates calculated from numerical simulation of the FPE, Eq. (19). Colored broken lines show perturbative exit rates according to Eqs. (44) and (54)-(56). Black solid lines denote the OM Lagrangian Eq. (55), from which the free-diffusion exit rate has already been subtracted so that in fact $\tilde{\alpha}^{(0)} / \tilde{\alpha}_{\text {free }}$ is plotted. Vertical dashed lines indicate the times $\tilde{t}=0.1,0.5,0.6$ used for subplots (a), (b), and (c).

In Figs. 3(a)-3(c), we compare the perturbative analytical probability density Eq. (53) to order $\epsilon^{5}$ with results from direct numerical solution of the FPE, Eq. (19). Figure 3 shows the probability density inside the tube at times (a) $\tilde{t}=0.1$, (b) $\tilde{t}=0.5$, and (c) $\tilde{t}=0.6$, as indicated by vertical dashed lines in Fig. 2. At each time we show results for radii $\epsilon=0.1$ (green), $\epsilon=0.5$ (blue), and $\epsilon=0.7$ (orange). Note that the intermediate tube radius $\epsilon \equiv R / L=0.5$ is in fact so large that during the ascent of the path $\varphi$ towards the barrier top there is a time at which the interval $B_{R}^{\varphi}(t)=[\varphi(t)-R, \varphi(t)+R]$ spans from the minimum $x=-L$ to the barrier top $x=0$.

For all times displayed, we observe that, while for $\epsilon=0.1$ and 0.5 numerical and perturbative results agree very well with each other, for the largest radius considered, $\epsilon=0.7$, clear deviations between the two are discernible. At the time $\tilde{t}=0.1$ considered in Fig. 3(a), the path $\varphi$ is close to the minimum at $x=-L$ and has a small velocity (see Fig. 2). While for the smallest radius $\epsilon=0.1$ the probability density is almost symmetric around $\tilde{x}=0$, indicating that the dynamics inside the tube is dominated by free diffusion, for $\epsilon=0.5$ and 0.7 the influence of the potential leads to a slight shift of the most probable position towards small negative values of $\tilde{x}$. The perturbative probability density for $\epsilon=0.7$ takes on negative values close to $\tilde{x}=-1$, which is clearly unphysical and signifies a breakdown of the perturbative results of order $\epsilon^{5}$. In Fig. 3(b) we show probability densities at time $\tilde{t}=0.5$, which according to Fig. 2 is when the path $\varphi$ traverses the barrier top. Despite the fact that at the maximum the potential $U$ is a symmetric function of $\tilde{x}$, all probability densities shown in Fig. 3(b) are tilted towards negative values of $\tilde{x}$. This is because the velocity of the path $\varphi$ leads to a fictitious force, as seen explicitly in Eq. (22); due to this fictitious force the symmetry of the potential $U$ is broken at the barrier top, which leads to the tilted probability densities observed in the figure. This effect is less pronounced at small $\epsilon$, where the dynamics inside the tube is dominated by free diffusion, as compared to the apparent deterministic force due to $U$ and $\dot{\varphi}$. In Fig. 3(c) we consider the time $\tilde{t}=0.6$, at which the path $\varphi$ descends from the barrier top towards the minimum at $x=L$ (see Fig. 2). Here we observe that, even though the force resulting from the potential $U$ pushes towards the positive $\tilde{x}$ direction, due to the velocity of the path $\varphi$ the apparent force Eq. (22) leads to a probability density that is still slightly tilted towards the negative $\tilde{x}$ direction, i.e., uphill in the potential-energy landscape.

In the Supplemental Material [43] we provide videos that show the full time evolution of the normalized probability density for radius $\epsilon=0.1,0.3,0.5$, and 0.7 . For $\epsilon=0.1$ and 0.3 , numerical and analytical results show perfect agreement throughout. Consistent with Fig. 3, for $\epsilon=0.5$ small deviations between numerical and analytical density are observed, and become most pronounced as the path $\varphi$ ascends the barrier $(\tilde{t} \approx 0.45)$; however, given the size of the tube the agreement between numerical and analytical probability density is 
remarkably good overall. For $\epsilon=0.7$ the breakdown of our perturbative results can be observed; the analytical probability takes on negative values and at times deviates considerably from the numerical data.

Overall, from Figs. 3(a)-3(c), and also the Supplemental Material [43], we conclude that for small to intermediate tube radius our analytic result Eq. (53) very well approximates the actual FP dynamics inside the tube.

\section{Exit rate from the tube}

Using the explicit expressions for the spectrum given in Appendix C, the expansion of the exit rate Eq. (44) in powers of $\epsilon$ is given by

$$
\begin{gathered}
\tilde{\alpha}_{\text {free }}=\frac{\pi^{2}}{4 \epsilon^{2}}, \\
\tilde{\alpha}^{(0)} \equiv \tilde{\mathcal{L}}_{\mathrm{OM}}=\frac{\tilde{E}_{1}^{2}}{4}-\tilde{E}_{2}, \\
\tilde{\alpha}^{(2)}=-\frac{\tilde{E}_{1} \dot{\tilde{E}}_{1}}{4}\left(1-\frac{8}{\pi^{2}}\right)+\frac{\dot{\tilde{E}}_{2}}{3}\left(1-\frac{9}{\pi^{2}}\right) \\
+\left(\frac{\tilde{E}_{1} \tilde{E}_{3}}{2}+\frac{\tilde{E}_{2}^{2}}{3}-2 \tilde{E}_{4}\right)\left(1-\frac{6}{\pi^{2}}\right),
\end{gathered}
$$

where the $\tilde{E}_{l} \equiv \tilde{E}_{l}(\tilde{t})$ are defined in Eq. (52), and a dot denotes a derivative with respect to $\tilde{t}$. We again consider barrier crossing in the double-well system, as defined in Eqs. (49) and (50) and illustrated in Fig. 2. In Figs. 3(d)-3(f), we compare numerically calculated exit rates to perturbative results obtained from Eqs. (44) and (54)-(56). In the plots the exit rate is shifted and rescaled according to

$$
\frac{\Delta \tilde{\alpha}_{\epsilon}^{\varphi}}{\tilde{\alpha}_{\text {free }}} \equiv \frac{\tilde{\alpha}_{\epsilon}^{\varphi}-\tilde{\alpha}_{\text {free }}}{\tilde{\alpha}_{\text {free }}},
$$

so that (i) the sign of a curve indicates whether the exit rate is enhanced or diminished as compared to the free-diffusion limit $\tilde{\alpha}_{\text {free }}$ and (ii) the magnitude yields the relative importance of the terms Eqs. (55) and (56) as compared to $\tilde{\alpha}_{\text {free }}$. Numerical data are shown as solid colored lines, and perturbative analytical results are given as broken colored lines. To gauge the importance of the quadratic correction Eq. (56) relative to the OM Lagrangian Eq. (55), we furthermore include plots of the latter as solid black lines in Figs. 3(d)-3(f). As detailed in Appendix C 5, we use as initial distribution $\tilde{P}_{i}$ for our simulations the instantaneous steady state of the FPE, so that there is no transient initial decay in our numerical data; a brief discussion of the transient effects of the initial condition on the exit rate is given in Appendix $\mathrm{C} 4$.

In Fig. 3(d) we consider the radius $\epsilon=0.1$. As can be seen, the numerical and analytical results agree perfectly with each other, and also with the OM Lagrangian Eq. (55). This means that the quadratic correction Eq. (56) is not yet relevant at this radius. Relative deviations from the free-diffusion exit rate $\tilde{\alpha}_{\text {free }}$ are less than $10 \%$ throughout, so that the exit rate is dominated by free diffusion. Figure 3(e) shows data for the intermediate radius $\epsilon=0.5$. Numerical and perturbative analytical results agree very well with each other, with minor deviations at $\tilde{t} \approx 0.5$. However, clear deviations between numerical data and the OM Lagrangian Eq. (55) are visible, meaning that the quadratic correction Eq. (56) to the exit rate is now relevant. The deviations between our perturbative/numerical results and OM theory are twofold. First, when the path is close to the minima, the OM action underestimates the true (numerical) exit rate. During these times, the numerical exit rate is rather insensitive to the exact position of the tube center within the well, because the rate limiting step to exit the tube is to climb the potential barrier, which is expected to be rather insensitive to the exact position of the tube center in the well. The second difference between our perturbative/numerical results and OM theory is that during barrier crossing the numerical exit rate is delayed as compared to the OM Lagrangian. From the magnitude of the rescaled exit rate Eq. (57), we conclude that for $\epsilon=0.5$ the free-diffusion exit rate is of the same order as the corrections Eqs. (55) and (56).

Figure 3(f) shows data for the largest radius $\epsilon=0.7$. Overall the perturbative result Eq. (44) still shows reasonable agreement with the numerical exit rate, which is surprising since the corresponding probability density at times deviates strongly from the numerical results [see Fig. 3(a) and the Supplemental Material [43]]. However, clear deviations between numerical and analytical exit rate can be discerned, most prominently during barrier crossing at $\tilde{t} \approx 0.5$. Numerical exit rate and OM Lagrangian Eq. (55) disagree considerably, showing the importance of the quadratic correction Eq. (56). During barrier crossing, the contributions to the exit rate from Eqs. (55) and (56) are about five times larger than the freediffusion exit rate $\tilde{\alpha}_{\text {free }}$.

In summary, Figs. 3(d)-3(f) show that our perturbative results Eqs. (44) and (54)-(56) describe the exit rate quantitatively up to a tube radius well comparable to the typical length scale of the potential $U$, and in particular beyond the regime where the OM Lagrangian is applicable.

To close this section, we illustrate how finite-radius exit rates obtained directly from measured trajectories compare to our perturbative analytical results. For this, we consider a tube radius $\epsilon=R / L=0.5$, as also discussed in Fig. 3(e). Figure 4 depicts the exit rate obtained directly from a large number of independent simulated time series. As Fig. 4 shows, the exit rate obtained directly from Langevin time series agrees well with our perturbative result Eq. (44), and shows clear deviations from the OM Lagrangian Eq. (55). This shows that it is possible to measure the exit rate for a finite-radius tube directly from time series, without fitting any model to the data. Note that since the FPE, Eq. (7), with absorbing boundary conditions is equivalent to the Langevin Eq. (1), with trajectories being discarded once they first cross the absorbing boundary, it is expected that Figs. 3(e) and 4 lead to the same conclusions; indeed, the agreement between numerical FP solution and results obtained from Langevin simulations is an important consistency check for our numerics. Apart from illustrating how our results directly connect to measured time series, the analysis based on Langevin trajectories also highlights two features that appear when extracting the exit rate from recorded data. First, since all Langevin simulations are initiated at $x=-L$, which can be thought of as a definite experimental initial condition, the exit rate shows a short transient relaxation period for times $\tilde{t} \lesssim 0.05$ (see Appendix C 4 for further discussion). Second, the number of trajectories inside the tube decreases over time, so that the statistics for 
calculating the exit rate become successively worse; this explains why the exit rate measured from Langevin trajectories starts to become noisy around $\tilde{t} \approx 0.5$.

\section{SUMMARY AND CONCLUSIONS}

In this paper we establish the finite-radius tubular ensemble, which consists of all stochastic trajectories that stay close to a smooth reference path $\varphi$, as a physically and mathematically useful concept to regularize and extend the path probabilities of individual stochastic trajectories. We in particular derive explicit expressions for the probability to observe any path of the tubular ensemble, thus generalizing the OM stochastic action. Our results have several important consequences.

From a mathematical perspective, we evaluate and study the probability $P\left(\mathcal{X}_{R}^{\varphi}\right)$, i.e., the probability that a stochastic trajectory stays close to a given smooth reference path, for finite radius $R$. We therefore focus on a measure, which is in contrast to previous work, which aimed to define probability densities on the space of all continuous paths, and therefore always involved the singular limit $R \rightarrow 0$ [29]. Compared to the approach to path probabilities via path integrals [23-28], an advantage of our approach is that at no point do we need to discretize time. Therefore, none of the technical/conceptual difficulties arising from different time-discretization schemes discussed in the literature arise [28,38,39]. Furthermore, in our theory smooth and nondifferentiable stochastic trajectories are cleanly disentangled. The former are used to parametrize a set (a moving ball with finite radius), and the latter are confined to this set.

In a sense, the approach used in the present paper is opposite to Freidlin-Wentzell theory [44]. While Freidlin and Wentzell also consider the tubular ensemble Eq. (2), they investigate the double limit of vanishing radius $R \rightarrow 0$ and temperature $1 / \beta=k_{\mathrm{B}} T \rightarrow 0$. Practically speaking, in their analysis the deterministic force in the Langevin Eq. (1) is assumed to be the dominant term. In our perturbative calculation at constant temperature, on the other hand, we perturb around the free-diffusion solution of the FPE, which means that in our analysis the random force term in the Langevin Eq. (1) is assumed to be the dominant term on short length scales. That random noise dominates over deterministic forces at short length and time scales is a basic feature of the Langevin equation and is in fact the reason why a typical realization of Eq. (1) is nowhere differentiable.

Our theory for the finite-radius tubular ensemble Eq. (2) is also highly relevant from a physical perspective. By establishing a direct relation between exit rate and stochastic action Lagrangian, we put the latter within reach of experiments. Indeed, substituting Eqs. (5) and (11) into Eq. (4), it follows that

$$
\mathcal{L}_{\mathrm{OM}}(\boldsymbol{\varphi}, \dot{\boldsymbol{\varphi}})-\mathcal{L}_{\mathrm{OM}}(\boldsymbol{\phi}, \dot{\boldsymbol{\phi}})=\lim _{R \rightarrow 0}\left[\alpha_{R}^{\varphi}-\alpha_{R}^{\phi}\right]
$$

While directly measuring the probability of an individual given path is experimentally unfeasible, simply because that probability is zero, directly measuring experimentally the probability for a trajectory to stay inside a moving ball with finite radius is possible with present-day technology $[9,45,46]$.

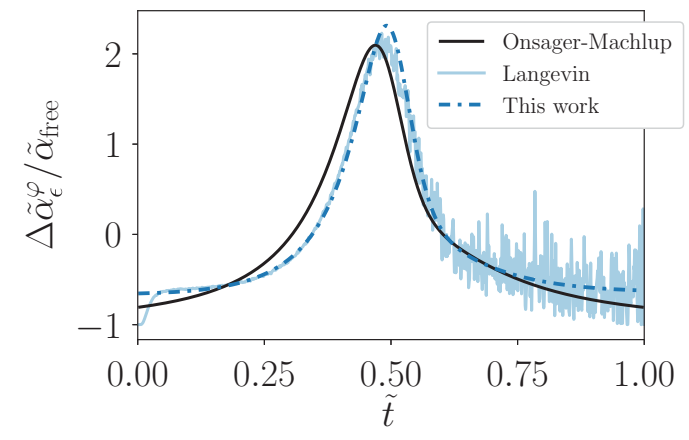

FIG. 4. Exit rate as measured directly from Langevin simulations for a tube of radius $\epsilon=R / L=0.5$. The black solid and blue dashdotted lines are replots of the corresponding lines in Fig. 3(e). The blue solid line represents the exit rate as estimated from simulated trajectories. For this, $2.4 \times 10^{6}$ independent Langevin simulations in the quartic double-well Eq. (49) with a simulation time step $\Delta \tilde{t}=$ $10^{-5}$ are performed, with each trajectory starting at $x=-L$. From these trajectories, the sojourn probability for a tube of radius $\epsilon=$ $R / L=0.5$ around a reference path $\varphi(t)$, as defined in Eq. (50), is evaluated directly, by counting which proportion of trajectories has never left the tube until any given time. Subsequently, the exit rate is numerically calculated via Eq. (41), and the result is smoothed using a moving average with a Hann window of width $\Delta \tilde{t}=0.003$. From this exit rate, finally, the free-diffusion exit rate Eq. (54) is subtracted, and the result is divided by the free-diffusion exit rate [see Eq. (57)].

This means the right-hand side of Eq. (58) can be measured for finite $R$, as was done in Fig. 4 , and then extrapolated to the limit $R \rightarrow 0$; this can be done without fitting a stochastic model to the time series. Equation (58) thus allows us to compare model-free measurements of exit rates (right-hand side of the equation) to theoretical predictions for the stochastic action Lagrangian (left-hand side of the equation). This will allow us to experimentally validate theoretical predictions for the stochastic action Lagrangian as a measure for relative path likelihoods. Equation (58) can furthermore serve as an operational and experimentally relevant definition for the action Lagrangian for other models of stochastic dynamics, for example, those used to describe active particles [47].

While irrelevant in the limit $R \rightarrow 0$, for finite tube radius it will be important to understand in more depth how transient effects due to the initial distribution $\boldsymbol{X}_{t_{i}} \sim P_{i}$ affect the sojourn probability. A basis for investigating such boundary effects is given by the full perturbative solution considered in Appendix B.

While we assume a smooth path $\varphi$, our derivation in fact only uses that it is twice differentiable. The first derivative $\dot{\varphi}$ emerges from applying the coordinate transformation Eq. (14) to the FPE [see Eq. (21)]. The second derivative enters because the FPE in terms of the instantaneous eigenbasis, Eq. (28), contains the time derivative of FP eigenfunctions; since these eigenfunctions depend on $\dot{\varphi}$, their derivative depends on $\ddot{\varphi}$. It will be interesting to extend our theory to reference paths $\varphi$ that are continuous, but not differentiable, such as realizations of the Langevin Eq. (1). A starting point for this would be to investigate how the FPE transforms under a nondifferentiable coordinate transformation [48]. 
Another possible extension of our theory is to include position-dependent diffusivity, i.e., to replace the constant diffusion coefficient $D$ by a function $D(\boldsymbol{x})$. Assuming that the diffusivity varies slowly along the tube, a first approximation is to simply replace $D$ by $D(\boldsymbol{x})$ in our results. In view of the exit rate Eq. (12), the sojourn probability is then given by

$$
\begin{aligned}
P_{R}^{\varphi}\left(t_{f}\right)= & \exp \left[-\frac{\tilde{\lambda}_{n}^{(0)}}{R^{2}} \int_{t_{i}}^{t_{f}} d t D(\varphi(t))\right. \\
& \left.-\int_{t_{i}}^{t_{f}} d t \mathcal{L}_{\mathrm{OM}}(t, \varphi(t), \dot{\varphi}(t))+\mathcal{O}\left(R^{2}\right)\right],
\end{aligned}
$$

where the diffusivity in the OM Lagrangian Eq. (6) is now evaluated at $D(\varphi(t))$. Equation (59) shows that for positiondependent diffusivity for small tube radius $R$ the leading-order difference in sojourn probabilities along two paths $\varphi$ and $\phi$ is the mean free-diffusion exit rate along the paths, and the OM action is now a sub-leading-order correction. Thus, in the limit $R \rightarrow 0$, instead of Eq. (6) one would rather want to consider an action

$$
S_{D}[\varphi] \equiv \int_{t_{i}}^{t_{f}} d t D(\varphi(t))
$$

to quantify physically observed relative path probabilities. Intuitively, a particle is more likely to diffuse away from a given reference path in a region with large diffusivity, as compared to a region with low diffusivity. In the mathematical literature the leading-order effect due to free diffusion, given by Eq. (60), is usually scaled away, essentially by introducing a position-dependent tube radius $R(x)$ such that $D(x) / R(x)^{2}$ is constant as a function of $\boldsymbol{x}$ [29]. Thus, before applying the OM theory in systems with position-dependent diffusivity, one should decide whether one wants to quantify relative path probabilities using a spatially constant threshold $R$, in which case one would want to use Eq. (60) as action, or using a varying threshold $R(\boldsymbol{x}) \sim \sqrt{D(\boldsymbol{x})}$, in which case the OM action is the leading-order difference in sojourn probabilities [29,37].

The present paper on the tubular ensemble Eq. (2) offers an intuitive picture on (relative) path probabilities for the Langevin Eq. (1), providing a physical approach to this hitherto rather technical subject. Since any question that can be posed for individual stochastic trajectories is straightforwardly extended to the tubular ensemble, and through that is made accessible to simulation or experiment, the theory presented here is expected to find many applications in the future. The results will be particularly useful for the field of stochastic thermodynamics, where the concept of individual trajectories, and ratios of their probabilities, is employed extensively $[18,19,47]$.

\section{ACKNOWLEDGMENTS}

We thank Prof. Mike Cates, Dr. Yongjoo Baek, Dr. Jules Guioth, Dr. Rob Jack, and Dr. Patrick Pietzonka for stimulating discussions; furthermore, we thank Dr. Kerstin Burghaus, Dr. Heather Partner, and Dr. Carlos Riofrío for helpful comments on the paper. Work was funded in part by the European Research Council under the European Union's Horizon 2020
Program, Grant No. 740269, and by an Early Career Grant to R.A. from the Isaac Newton Trust.

\section{APPENDIX A: PERTURBATIVE SPECTRUM OF THE $N$-DIMENSIONAL FPE}

\section{Perturbation theory}

In the present Appendix, we perturbatively solve the eigenvalue Eq. (25) up to order $\epsilon^{2}$. For this, we first expand the right-hand side of the equation as a power series in $\epsilon$.

\section{a. Taylor expansion of the force}

The multidimensional Taylor expansion of the force $\boldsymbol{F}$ around the tube center $\varphi(t)$ is given by

$$
\begin{aligned}
\boldsymbol{F}(\boldsymbol{x}, t)= & \left.\sum_{k=0}^{\infty} \frac{1}{k !} \sum_{\alpha_{1}, \ldots, \alpha_{k}=1}^{N} \frac{\partial^{k} \boldsymbol{F}}{\partial x_{\alpha_{1}} \ldots \partial x_{\alpha_{k}}}\right|_{(\boldsymbol{\varphi}(t), t)} \\
& \times[\boldsymbol{x}-\boldsymbol{\varphi}(t)]_{\alpha_{1}} \ldots[\boldsymbol{x}-\boldsymbol{\varphi}(t)]_{\alpha_{k}},
\end{aligned}
$$

where $[\boldsymbol{x}-\boldsymbol{\varphi}(t)]_{\alpha_{i}} \equiv x_{\alpha_{i}}-\varphi_{\alpha_{i}}(t)$ is the $\alpha_{i}$ th component of the vector $\boldsymbol{x}-\boldsymbol{\varphi}(t)$. Substituting Eq. (A1) into the definition of the dimensionless force Eq. (17) and using Eq. (14), we obtain that

$$
\begin{aligned}
\tilde{\boldsymbol{F}}_{\mathrm{app}}(\tilde{\boldsymbol{x}}, \tilde{t}) & =-\sum_{k=1}^{\infty} \epsilon^{k-1} k \sum_{\alpha_{1}, \ldots, \alpha_{k-1}=1}^{N} \tilde{\boldsymbol{E}}_{k, \alpha_{1} \ldots \alpha_{k-1}}(\tilde{t}) \tilde{x}_{\alpha_{1}} \ldots \tilde{x}_{\alpha_{k-1}} \\
& \equiv-\sum_{k=1}^{\infty} \epsilon^{k-1} k \tilde{\boldsymbol{E}}_{k, \boldsymbol{\alpha}}(\tilde{t}) \tilde{x}_{\boldsymbol{\alpha}}
\end{aligned}
$$

where we use the Einstein sum convention for the indices $\boldsymbol{\alpha} \equiv\left(\alpha_{1}, \ldots, \alpha_{k-1}\right)$, we abbreviate $\tilde{x}_{\boldsymbol{\alpha}} \equiv \tilde{x}_{\alpha_{1}} \ldots \tilde{x}_{\alpha_{k-1}}$, and the vector-valued $(k-1)$-multilinear form $\tilde{\boldsymbol{E}}_{k}$ is defined as

$$
\tilde{\boldsymbol{E}}_{k, \alpha_{1} \ldots \alpha_{k-1}}(\tilde{t}) \equiv-\left.\frac{1}{k !} L^{k} \beta \frac{\partial^{k-1} \boldsymbol{F}}{\partial x_{\alpha_{1}} \ldots \partial x_{\alpha_{k-1}}}\right|_{(\boldsymbol{\varphi}(t), t)}+\delta_{k, 1} \dot{\tilde{\boldsymbol{\varphi}}}(\tilde{t}),
$$

where dimensionless quantities (as indicated by a tilde) and quantities with physical dimensions are related via Eqs. (14) and (18). Note that if the derivatives of the force commute, e.g., if the force is a smooth function of position for a time $t$, then $\tilde{\boldsymbol{E}}_{k}$ is symmetric in the $\left(\alpha_{1}, \ldots, \alpha_{k-1}\right)$. If the force is locally given by a potential $U$ as $\boldsymbol{F}=-\nabla U$, then the $j$ th vector component of $\tilde{\boldsymbol{E}}_{k}$ is given by

$$
\begin{aligned}
\tilde{E}_{k, \alpha_{1} \ldots \alpha_{k-1}}^{j}(\tilde{t}) \equiv & \left.\frac{1}{k !} L^{k} \beta \frac{\partial^{k} U}{\partial x_{\alpha_{1}} \ldots \partial x_{\alpha_{k-1}} \partial x_{j}}\right|_{(\varphi(t), t)} \\
& +\delta_{k, 1} \dot{\tilde{\varphi}}_{j}(\tilde{t}),
\end{aligned}
$$

so that Eq. (A3) is a multivariate Taylor expansion of the dimensionless potential $\tilde{U}=\beta U+\tilde{\boldsymbol{x}} \cdot \dot{\tilde{\boldsymbol{\varphi}}}$ around the tube center $\tilde{\boldsymbol{x}}=0$. Consequently, in that case the un-normalized instantaneous steady-state distribution inside the tube is given by

$$
\tilde{\rho}_{\mathrm{SS}}(\tilde{\boldsymbol{x}}, \tilde{t})=\exp \left[-\sum_{k=1}^{\infty} \epsilon^{k} \sum_{\alpha_{1}, \ldots, \alpha_{k}=1}^{N} \tilde{\boldsymbol{E}}_{k, \alpha_{1}, \ldots, \alpha_{k-1}}^{\alpha_{k}} \tilde{x}_{\alpha_{1}} \ldots \tilde{x}_{\alpha_{k}}\right] .
$$




\section{b. Hierarchy of equations for the spectrum}

Inserting the power series Eq. (A3) into the eigenvalue Eq. (25), we obtain

$$
\tilde{\nabla}^{2} \tilde{\rho}_{n}+\sum_{k=1}^{\infty} k \epsilon^{k}\left(\tilde{\boldsymbol{E}}_{k, \boldsymbol{\alpha}} \cdot \tilde{\nabla}\right)\left(\tilde{x}_{\boldsymbol{\alpha}} \tilde{\rho}_{n}\right)=-\tilde{\lambda}_{n} \tilde{\rho}_{n},
$$

where the dot denotes a scalar product and $\tilde{\nabla}$ denotes the gradient operator with vector components $\tilde{\nabla}_{i} \equiv \partial / \partial \tilde{x}_{i}$.

Expanding both the instantaneous eigenvalues and eigenfunctions as power series in $\epsilon$, as defined in Eq. (29), substituting these into Eq. (A7), and demanding that the resulting equation hold at each power $\epsilon^{k}$, we obtain a hierarchy of equations which for the $n$th eigenvalue/eigenfunction pair at order $\epsilon^{k}$ read

$$
\begin{aligned}
& \tilde{\nabla}^{2} \tilde{\rho}_{n}^{(k)}+\tilde{\lambda}_{n}^{(0)} \tilde{\rho}_{n}^{(k)} \\
& =-\sum_{l=1}^{k} \tilde{\lambda}_{n}^{(l)} \tilde{\rho}_{n}^{(k-l)}-\sum_{l=1}^{k} l\left(\tilde{\boldsymbol{E}}_{l, \boldsymbol{\alpha}} \cdot \tilde{\nabla}\right)\left(\tilde{x}_{\boldsymbol{\alpha}} \tilde{\rho}_{n}^{(k-l)}\right),
\end{aligned}
$$

where we use the convention that for $k=0$ the sums on the right-hand side are zero. For the absorbing boundary conditions to be fulfilled independently of $\epsilon$, they need to hold at each order separately, so that for all $k \in\{0,1,2, \ldots\}$ we have

$$
\tilde{\rho}_{n}^{(k)}(\tilde{\boldsymbol{x}}, \tilde{t})=0 \quad \forall \tilde{x} \in \partial \tilde{\boldsymbol{B}} \equiv\{\tilde{\boldsymbol{x}} \mid\|\tilde{\boldsymbol{x}}\|=1\} .
$$

While any solution to Eqs. (A8) and (A9) can be used in practice for the spectrum, the solution to these equations is not unique. To fix the solution uniquely, we introduce a normalization condition $\left\langle\tilde{\rho}_{n}, \tilde{\rho}_{n}\right\rangle=1$. Inserting the power-series expansion Eq. (29) for the eigenfunction into this normalization condition, and demanding that the condition hold at each power of $\epsilon$, we obtain for $k=0$ that

$$
\int_{\tilde{B}} d^{N} \tilde{\boldsymbol{x}} \tilde{\rho}_{n}^{(0)} \tilde{\rho}_{n}^{(0)}=1,
$$

while for $k \geqslant 1$ we have that

$$
\begin{aligned}
& \int_{\tilde{B}} d^{N} \tilde{\boldsymbol{x}} \tilde{\rho}_{n}^{(k)} \tilde{\rho}_{n}^{(0)} \\
& \quad=-\frac{1}{2} \sum_{l=0}^{k-1} \sum_{m=0}^{k-\max \{1, l\}} \int_{\tilde{B}} d^{N} \tilde{\boldsymbol{x}} \tilde{\rho}_{n}^{(l)} \tilde{\rho}_{n}^{(m)}\left(\tilde{\rho}_{\mathrm{SS}}^{-1}\right)^{(k-l-m)}
\end{aligned}
$$

where we use the convention that for $k=1$ the sum on the right-hand side is zero and the expansion of $\tilde{\rho}_{\mathrm{SS}}^{-1}$ in powers of $\epsilon$ is discussed in Appendix A 2. Note that for any $k$ there only appear perturbation terms $\tilde{\rho}_{n}^{(l)}$ with $l<k$ on the right-hand side of Eq. (A11).

Equations (A8)-(A11) constitute a closed system of equations that can be solved recursively to obtain the spectrum to arbitrary order.

At order $k=0$, the right-hand side of Eq. (A8) vanishes, so that the equation is reduced to the eigenvalue equation of the Laplace operator. Thus, $\tilde{\lambda}_{n}^{(0)}$ and $\tilde{\rho}_{n}^{(0)}$ comprise the spectrum of the Laplace operator with absorbing boundary conditions on a unit ball, where we assume that $\tilde{\rho}_{n}^{(0)}$ has been normalized according to Eq. (A10).
Assuming the spectrum has been obtained up to order $k-1$, the contribution at order $k$ is calculated as follows. An equation for $\tilde{\lambda}_{n}^{(k)}$ is obtained by multiplying Eq. (A8) with $\tilde{\rho}_{n}^{(0)}$, and subsequently integrating over $\tilde{\boldsymbol{x}}$. Upon integrating the result by parts and using the absorbing boundary conditions Eq. (A9), it follows that the equation is in fact independent of $\tilde{\rho}_{n}^{(k)}$ and can be solved directly for $\tilde{\lambda}_{n}^{(k)}$, leading to

$$
\begin{aligned}
\tilde{\lambda}_{n}^{(k)}= & -\sum_{l=1}^{k-1} \tilde{\lambda}_{n}^{(l)} \int_{-1}^{1} d \tilde{x} \tilde{\rho}_{n}^{(0)} \tilde{\rho}_{n}^{(k-l)} \\
& -\sum_{l=1}^{k} l \int_{\tilde{B}} d^{N} \tilde{\boldsymbol{x}} \tilde{\rho}_{n}^{(0)}\left(\tilde{\boldsymbol{E}}_{l, \boldsymbol{\alpha}} \cdot \tilde{\nabla}\right)\left(\tilde{x}_{\boldsymbol{\alpha}} \tilde{\rho}_{n}^{(k-l)}\right),
\end{aligned}
$$

where we used the normalization condition Eq. (A10) for $\tilde{\rho}_{n}^{(0)}$. Since the right-hand side only depends on $\tilde{\lambda}_{n}^{(l)}$ and $\tilde{\rho}_{n}^{(l)}$ with $l<k$, this equation can be used to calculate the order $k$ eigenvalue contribution in terms of the lower-order contributions.

Once $\tilde{\lambda}_{n}^{(k)}$ has been obtained via Eq. (A12), the right-hand side of Eq. (A8) is known, so that to obtain $\tilde{\rho}_{n}^{(k)}$ the inhomogeneous Helmholtz Eq. (A8) with boundary conditions Eq. (A9) has to be solved. While in general this can be done using the corresponding Green's function, we calculate the spectrum to order $\epsilon^{2}$ directly using a simple ansatz below. Before that, however, we establish some general properties of the spectrum which follow from parity symmetry.

\section{c. Parity properties of the spectrum}

We introduce the parity operator $\tilde{\mathcal{P}}$, defined by its action on a function $f$ as

$$
(\tilde{\mathcal{P}} f)(\tilde{\boldsymbol{x}}) \equiv f(-\tilde{\boldsymbol{x}})
$$

Consequently, for products of functions $f$ and $g$, it holds that $\tilde{\mathcal{P}}(f g)=(\tilde{\mathcal{P}} f)(\tilde{\mathcal{P}} g)$, and for the gradient we have $\tilde{\mathcal{P}} \tilde{\nabla}=$ $-\tilde{\nabla}$. Therefore the operator $\tilde{\mathcal{P}}$ commutes with the Laplacian, $\tilde{\mathcal{P}} \tilde{\nabla}^{2}=\tilde{\nabla}^{2} \tilde{\mathcal{P}}$, so that we can assume that the eigenfunctions $\tilde{\rho}_{n}^{(0)}$ of the Laplacian diagonalize $\tilde{\nabla}^{2}$ and $\tilde{\mathcal{P}}$ simultaneously, so that

$$
\tilde{\mathcal{P}} \tilde{\rho}_{n}^{(0)}=p_{n} \tilde{\rho}_{n}^{(0)},
$$

with $p_{n} \in\{-1,1\}$

Via induction in $k$ it follows from Eqs. (A8), (A9), (A12), and (A14) that

$$
\tilde{\lambda}_{n}^{(k)}=0 \text { for } k \text { odd }
$$

and furthermore that

$$
\tilde{\mathcal{P}} \tilde{\rho}_{n}^{(k)}=(-1)^{k} p_{n} \tilde{\rho}_{n}^{(k)} .
$$

Thus, $\tilde{\rho}_{n}^{(k)}$ has the same parity as $\tilde{\rho}_{n}^{(0)}$ if $k$ is even, and the opposite parity as $\tilde{\rho}_{n}^{(0)}$ if $k$ is odd.

We now calculate the lowest-order contributions to the $N$-dimensional FP spectrum; higher-order results for onedimensional systems are given in Appendix $\mathrm{C} 1$. 


\section{d. Order $\epsilon^{1}$ contribution to the spectrum}

For $k=1$, Eq. (A15) yields $\tilde{\lambda}_{n}^{(1)}=0$. Substituting this into Eq. (A8) for $k=1$, we obtain

$$
\tilde{\nabla}^{2} \tilde{\rho}_{n}^{(1)}+\tilde{\lambda}_{n}^{(0)} \tilde{\rho}_{n}^{(1)}=-\left(\tilde{\boldsymbol{E}}_{1} \cdot \nabla\right) \tilde{\rho}_{n}^{(0)} .
$$

As can be verified by direct substitution, a solution to this inhomogeneous Helmholtz equation is given by

$$
\tilde{\rho}_{n}^{(1)}(\tilde{\boldsymbol{x}}, \tilde{t})=-\frac{1}{2}\left[\tilde{\boldsymbol{E}}_{1}(\tilde{t}) \cdot \tilde{\boldsymbol{x}}\right] \tilde{\rho}_{n}^{(0)}(\tilde{\boldsymbol{x}}, \tilde{t}),
$$

where the dot denotes the standard Euclidean inner product between the two $N$-dimensional vectors $\tilde{\boldsymbol{E}}_{1}(\tilde{t})$ and $\tilde{\boldsymbol{x}}$. Because $\tilde{\rho}_{n}^{(0)}$ vanishes on $\partial \tilde{B}$, the result Eq. (A18) fulfills the boundary condition Eq. (A9). For $k=1$, the normalization condition Eq. (A11) is fulfilled by Eq. (A18), because upon substitution of Eq. (A18) the integrand in Eq. (A11) has odd parity, while the integration domain is symmetric with respect to a parity transformation. With Eqs. (A15) and (A18), we thus have the order $\epsilon^{1}$ contribution to the $N$-dimensional FP spectrum.

\section{e. Order $\epsilon^{2}$ contribution to the spectrum}

For $k=2$, Eq. (A12) becomes

$$
\begin{aligned}
\tilde{\lambda}_{n}^{(2)}= & -\int_{\tilde{B}} d^{N} \tilde{\boldsymbol{x}} \tilde{\rho}_{n}^{(0)}\left(\tilde{\boldsymbol{E}}_{1} \cdot \tilde{\boldsymbol{\nabla}}\right)\left(\tilde{\rho}_{n}^{(1)}\right) \\
& -2 \int_{\tilde{B}} d^{N} \tilde{\boldsymbol{x}} \tilde{\rho}_{n}^{(0)}\left(\tilde{\boldsymbol{E}}_{2, \alpha} \cdot \tilde{\boldsymbol{\nabla}}\right)\left(\tilde{x}_{\alpha} \tilde{\rho}_{n}^{(0)}\right),
\end{aligned}
$$

where we use $\tilde{\lambda}_{n}^{(1)}=0$. Substituting Eq. (A18) into Eq. (A19), and performing the integrals, we obtain the second correction for the eigenvalue as

$$
\tilde{\lambda}_{n}^{(2)}=\frac{\tilde{\boldsymbol{E}}_{1}^{2}}{4}-\operatorname{tr}\left(\tilde{\boldsymbol{E}}_{2}\right)
$$

where

$$
\left(\tilde{\boldsymbol{E}}_{2, \alpha} \cdot \tilde{\nabla}\right)\left(\tilde{x}_{\alpha}\right)=\sum_{i=1}^{N} \tilde{\boldsymbol{E}}_{2, i}^{i} \equiv \operatorname{tr}\left(\tilde{\boldsymbol{E}}_{2}\right) .
$$

Substituting the definition of $\tilde{\boldsymbol{E}}_{k, \boldsymbol{\alpha}}$, Eq. (A4), and using Eqs. (17) and (18), it follows that

$$
\tilde{\lambda}_{n}^{(2)}=\tau_{D}\left[\frac{\left(\left.D \beta \boldsymbol{F}\right|_{\varphi}-\dot{\varphi}\right)^{2}}{4 D}+\left.\frac{D \beta}{2}(\nabla \cdot \boldsymbol{F})\right|_{\varphi}\right],
$$

which is the OM stochastic action in units of $1 / \tau_{D} \equiv D / L^{2}$. To calculate $\tilde{\rho}_{n}^{(2)}$, we insert Eqs. (A15), (A18), and (A20) into the right-hand side of Eq. (A8) (with $k=2$ ), resulting in

$$
\begin{aligned}
\tilde{\nabla}^{2} \tilde{\rho}_{n}^{(2)}+\tilde{\lambda}_{n}^{(0)} \tilde{\rho}_{n}^{(2)}= & {\left[\frac{\tilde{\boldsymbol{E}}_{1}^{2}}{4}-\operatorname{tr}\left(\tilde{\boldsymbol{E}}_{2}\right)\right] \tilde{\rho}_{n}^{(0)} } \\
& +\left[\frac{1}{2}\left(\tilde{\boldsymbol{E}}_{1} \cdot \tilde{\boldsymbol{x}}\right) \tilde{\boldsymbol{E}}_{1}-2 \tilde{\boldsymbol{E}}_{2, \boldsymbol{\alpha}} \tilde{x}_{\boldsymbol{\alpha}}\right] \cdot \tilde{\nabla} \tilde{\rho}_{n}^{(0)}
\end{aligned}
$$

This equation can be solved directly for the case where the force inside the tube is given as the gradient of an instantaneous potential, $\boldsymbol{F}=-\nabla U$. According to Eq. (A5), in that case the two-tensor (or vector-valued one form) $\tilde{\boldsymbol{E}}_{2}$ is symmetric, i.e., we have $\tilde{E}_{2, i}^{j}=\tilde{E}_{2, j}^{i}$, and direct substitution shows that Eq. (A23) is solved by

$$
\tilde{\rho}_{n}^{(2)}=\frac{1}{2}\left[\frac{\left(\tilde{\boldsymbol{E}}_{1} \cdot \tilde{\boldsymbol{x}}\right)^{2}}{4}-\tilde{\boldsymbol{x}} \cdot \tilde{\boldsymbol{E}}_{2, \alpha} \tilde{x}_{\alpha}\right] \tilde{\rho}_{n}^{(0)},
$$

which fulfills both the normalization condition Eq. (A11) and the boundary conditions Eq. (A9) (note that $\tilde{\rho}_{n}^{(0)}$ vanishes on $\partial \tilde{B})$. The solution Eq. (A24) is also valid if $\tilde{\rho}_{n}^{(0)}$ is radially symmetric, as is the case for $n=1$. In that case $\tilde{\rho}_{n}^{(0)}$ depends on $\tilde{\boldsymbol{x}}$ only via $\|\tilde{\boldsymbol{x}}\|$, and consequently there is a scalar function $f$ such that $\tilde{\nabla} \tilde{\rho}_{n}^{(0)}=f(\|\tilde{\boldsymbol{x}}\|) \tilde{\boldsymbol{x}}$. Using this, it is readily verified that Eq. (A24) is a solution to Eq. (A23).

\section{f. Order $\epsilon^{3}$ contribution to the eigenvalue}

According to Eq. (A15), we have $\tilde{\lambda}_{n}^{(3)}=0$.

\section{Parity properties of the reflecting-boundary steady state}

In the present section we discuss the perturbative calculation and parity properties of both the steady state $\tilde{\rho}_{\text {SS }}$ and its multiplicative inverse $\tilde{\rho}_{\text {SS }}^{-1} \equiv 1 / \tilde{\rho}_{\text {SS }}$.

\section{a. Perturbative calculation of $\tilde{\rho}_{\mathrm{SS}}$}

According to Eq. (21), the instantaneous steady state $\tilde{\rho}_{\mathrm{SS}}$ is the solution of the boundary value problem

$$
\tilde{\nabla}^{2} \tilde{\rho}_{\mathrm{SS}}-\epsilon \tilde{\nabla} \cdot\left[\tilde{\boldsymbol{F}}_{\mathrm{app}} \tilde{\rho}_{\mathrm{SS}}\right]=0,
$$

with boundary condition

$$
\left.\hat{\mathbf{n}} \cdot \tilde{\mathbf{j}}_{\mathrm{ss}}\right|_{\partial \tilde{B}}=0,
$$

where $\tilde{\mathbf{j}}_{\mathrm{ss}} \equiv-\tilde{\nabla} \tilde{\rho}_{\mathrm{SS}}+\epsilon \tilde{\boldsymbol{F}}_{\mathrm{app}} \tilde{\rho}_{\mathrm{SS}}$, where $\hat{\boldsymbol{n}}$ is the unit normal vector on $\tilde{B}$, and where $\tilde{\boldsymbol{F}}_{\text {app }}=\tilde{\boldsymbol{F}}-\dot{\tilde{\boldsymbol{\varphi}}}$, as defined in Eq. (22).

If the force $\tilde{\boldsymbol{F}}$ originates from a potential, $\tilde{\boldsymbol{F}}=-\tilde{\nabla} \tilde{U}$, then the (un-normalized) instantaneous steady state is a Boltzmann distribution [see Eqs. (27) and (A6)]. Using the Taylor expansion of the exponential function, an expansion in powers of $\epsilon$ for $\tilde{\rho}_{\mathrm{SS}}$ is then obtained from Eq. (A6).

We now discuss how to perturbatively calculate $\tilde{\rho}_{\text {SS }}$ for the general case, in which the force $\tilde{\boldsymbol{F}}$ need not have an instantaneous potential inside the tube. Substituting into Eq. (A25) the power-series expansion Eq. (A3) of $\tilde{\boldsymbol{F}}$, we obtain

$$
\tilde{\nabla}^{2} \tilde{\rho}_{\mathrm{SS}}+\sum_{k=1}^{\infty} k \epsilon^{k}\left(\tilde{\boldsymbol{E}}_{k, \boldsymbol{\alpha}} \cdot \tilde{\nabla}\right)\left(\tilde{x}_{\boldsymbol{\alpha}} \tilde{\rho}_{\mathrm{SS}}\right)=0,
$$

where the dot denotes the standard Euclidean inner product. Expanding the instantaneous steady state as power series in $\epsilon$,

$$
\tilde{\rho}_{\mathrm{SS}}=\sum_{k=0}^{\infty} \epsilon^{k} \tilde{\rho}_{\mathrm{SS}}^{(k)},
$$

substituting this expansion into Eq. (A27), and demanding that the resulting equation hold at each power $\epsilon^{k}$, we obtain a hierarchy of equations which at order $\epsilon^{k}$ reads

$$
\tilde{\nabla}^{2} \tilde{\rho}_{\mathrm{SS}}^{(k)}=-\sum_{l=1}^{k} l\left(\tilde{\boldsymbol{E}}_{l, \boldsymbol{\alpha}} \cdot \tilde{\nabla}\right)\left(\tilde{x}_{\boldsymbol{\alpha}} \tilde{\rho}_{\mathrm{SS}}^{(k-l)}\right),
$$

where we use the convention that for $k=0$ the sum on the right-hand side is zero. Inserting the power-series expansions 
Eq. (A3) and (A28) into the boundary condition Eq. (A26), and demanding that the resulting equation be fulfilled at each power $\epsilon^{k}$, we obtain

$$
(\hat{\mathbf{n}} \cdot \tilde{\nabla}) \tilde{\rho}_{\mathrm{SS}}^{(k)}=-\sum_{l=1}^{k} l\left(\hat{\mathbf{n}} \cdot \tilde{\boldsymbol{E}}_{l, \boldsymbol{\alpha}}\right) \tilde{x}_{\boldsymbol{\alpha}} \tilde{\rho}_{\mathrm{SS}}^{(k-l)}
$$

where $k \geqslant 0$ and we use the convention that for $k=0$ the sum on the right-hand side is zero.

While at order $\epsilon^{0}$ the (un-normalized) solution to Eqs. (A29) and (A30) is simply given by $\tilde{\rho}_{\mathrm{SS}}^{(0)}=1$, for $k \geqslant 1$ the equations have to be solved recursively, similar to the spectrum in Appendix A1. The resulting corrections at order 1 and 2 are

$$
\begin{gathered}
\tilde{\rho}_{\mathrm{SS}}^{(1)}=-\tilde{\boldsymbol{E}}_{1} \cdot \tilde{\boldsymbol{x}}, \\
\tilde{\rho}_{\mathrm{SS}}^{(2)}=\frac{1}{2}\left(\tilde{\boldsymbol{E}}_{1} \cdot \tilde{\boldsymbol{x}}\right)^{2}-\tilde{\boldsymbol{x}} \cdot \tilde{\boldsymbol{E}}_{2, \alpha} \tilde{x}_{\alpha} .
\end{gathered}
$$

Note that in Eq. (A32) only the symmetric part of the twotensor (or vector-valued one form) $\tilde{\boldsymbol{E}}_{2}$ contributes.

\section{b. Parity properties of the $\tilde{\rho}_{\mathrm{SS}}^{(k)}$}

Similar to the parity properties of the FP spectrum, via induction in $k$ it can be shown that

$$
\tilde{\mathcal{P}} \tilde{\rho}_{\text {SS }}^{(k)}=(-1)^{k} \tilde{\rho}_{\text {SS }}^{(k)},
$$

where the parity operator $\tilde{\mathcal{P}}$ is defined in Eq. (A13).

\section{c. Perturbative calculation and parity properties of $\tilde{\rho}_{\mathrm{SS}}^{-1}$}

By definition of the inverse, it holds that

$$
\tilde{\rho}_{\mathrm{SS}} \tilde{\rho}_{\mathrm{SS}}^{-1}=1 .
$$

Substituting the power-series expansion Eq. (A28) of $\tilde{\rho}_{\text {SS }}$ and the expansion

$$
\tilde{\rho}_{\mathrm{SS}}^{-1}=\sum_{k=0}^{\infty} \epsilon^{k}\left(\tilde{\rho}_{\mathrm{SS}}^{-1}\right)^{(k)},
$$

into Eq. (A34), and demanding that the equation hold at any order of $\epsilon$, we obtain a recursive system of equations for the expansion of $\tilde{\rho}_{\text {SS }}^{-1}$ given by

$$
\begin{gathered}
\left(\tilde{\rho}_{\mathrm{SS}}^{-1}\right)^{(0)}=1, \\
\left(\tilde{\rho}_{\mathrm{SS}}^{-1}\right)^{(k)}=-\sum_{l=1}^{n} \tilde{\rho}_{\mathrm{SS}}^{(l)}\left(\tilde{\rho}_{\mathrm{SS}}^{-1}\right)^{(k-l)},
\end{gathered}
$$

where in Eq. (A36) we use that $\tilde{\rho}_{\mathrm{SS}}^{(0)}=1$. Using Eqs. (A31) and (A32), it follows from Eq. (A37) that

$$
\begin{gathered}
\left(\tilde{\rho}_{\mathrm{SS}}^{-1}\right)^{(1)}=\tilde{\boldsymbol{E}}_{1} \cdot \tilde{\boldsymbol{x}}, \\
\left(\tilde{\rho}_{\mathrm{SS}}^{-1}\right)^{(2)}=\frac{1}{2}\left(\tilde{\boldsymbol{E}}_{1} \cdot \tilde{\boldsymbol{x}}\right)^{2}+\tilde{\boldsymbol{x}} \cdot \tilde{\boldsymbol{E}}_{2, \alpha} \tilde{x}_{\alpha} .
\end{gathered}
$$

Note that in Eq. (A39) only the symmetric part of the twotensor $\tilde{\boldsymbol{E}}_{2}$ contributes.

According to Eq. (A36), the parity of $\left(\tilde{\rho}_{\mathrm{SS}}^{-1}\right)^{(0)}$ is 1 . Using induction, and applying the parity operator to Eq. (A37), it furthermore follows that

$$
\tilde{\mathcal{P}}\left[\left(\tilde{\rho}_{\mathrm{SS}}^{-1}\right)^{(k)}\right]=(-1)^{k}\left(\tilde{\rho}_{\mathrm{SS}}^{-1}\right)^{(k)}
$$

for all $k$.

\section{Properties of power-series expansions derived from parity}

We now derive properties of some power-series expansions used in the main text.

\section{a. Integral over FP eigenfunctions}

We consider

$$
\tilde{\mathcal{I}}_{n} \equiv \int_{\tilde{B}} d^{N} \tilde{\boldsymbol{x}} \tilde{\rho}_{n},
$$

which we expand in a power series

$$
\tilde{\mathcal{I}}_{n} \equiv \sum_{k=0}^{\infty} \epsilon^{k} \tilde{\mathcal{I}}_{n}^{(k)}
$$

with $\tilde{\mathcal{I}}_{n}^{(k)}$ defined by Eq. (48). The integral on the right-hand side of Eq. (48) vanishes if $\tilde{\rho}_{n}^{(k)}$ has odd parity. According to Eq. (A16), we thus have

$$
\tilde{\mathcal{I}}_{n}^{(k)}=0 \text { if }\left\{\begin{array}{l}
k \text { odd and } p_{n}=1, \\
k \text { even and } p_{n}=-1 .
\end{array}\right.
$$

In particular, since the lowest eigenfunction of the Laplace operator (inside a unit ball and with absorbing boundary conditions) is even, we have

$$
\tilde{\mathcal{I}}_{1}=\tilde{\mathcal{I}}_{1}^{(0)}+\epsilon^{2} \tilde{\mathcal{I}}_{1}^{(2)}+\epsilon^{4} \tilde{\mathcal{I}}_{1}^{(4)}+\mathcal{O}\left(\epsilon^{6}\right) .
$$

\section{b. Inner product of FP eigenfunctions}

We consider

$$
\left\langle\tilde{\rho}_{n}, \tilde{\rho}_{m}\right\rangle=\int_{\tilde{B}} d^{N} \tilde{\boldsymbol{x}} \tilde{\rho}_{n} \tilde{\rho}_{m} \tilde{\rho}_{\mathrm{SS}}^{-1}
$$

[see Eq. (26)]. The power-series expansion of this inner product is given by

$$
\left\langle\tilde{\rho}_{n}, \tilde{\rho}_{m}\right\rangle=\sum_{l=0}^{\infty} \epsilon^{l}\left\langle\tilde{\rho}_{n}, \tilde{\rho}_{m}\right\rangle^{(l)},
$$

where

$$
\left\langle\tilde{\rho}_{n}, \tilde{\rho}_{m}\right\rangle^{(l)}=\sum_{\substack{i, j, k \geqslant 0 \\ i+j+k=l}} \int_{\tilde{B}} d^{N} \tilde{\boldsymbol{x}} \tilde{\rho}_{n}^{(i)} \tilde{\rho}_{m}^{(j)}\left(\tilde{\rho}_{\mathrm{SS}}^{-1}\right)^{(k)}
$$

with the power-series expansions Eq. (29) and (A35). If the integrand has odd parity, the integral on the right-hand side vanishes; applying the parity operator to the integrand we calculate

$$
\tilde{\mathcal{P}}\left[\tilde{\rho}_{n}^{(i)} \tilde{\rho}_{m}^{(j)}\left(\tilde{\rho}_{\mathrm{SS}}^{-1}\right)^{(k)}\right]=p_{n} p_{m}(-1)^{l}\left[\tilde{\rho}_{n}^{(i)} \tilde{\rho}_{m}^{(j)}\left(\tilde{\rho}_{\mathrm{SS}}^{-1}\right)^{(k)}\right],
$$

where we use Eqs. (A16) and (A40), and $i+j+k=l$. Thus, we have

$$
\left\langle\tilde{\rho}_{n}, \tilde{\rho}_{m}\right\rangle^{(l)}=0 \text { if }\left\{\begin{array}{l}
l \text { odd and } p_{n} p_{m}=1, \\
l \text { even and } p_{n} p_{m}=-1 .
\end{array}\right.
$$

In particular, we have

$$
\left\langle\tilde{\rho}_{n}, \tilde{\rho}_{n}\right\rangle=\left\langle\tilde{\rho}_{n}, \tilde{\rho}_{n}\right\rangle^{(0)}+\epsilon^{2}\left\langle\tilde{\rho}_{n}, \tilde{\rho}_{n}\right\rangle^{(2)}+\epsilon^{4}\left\langle\tilde{\rho}_{n}, \tilde{\rho}_{n}\right\rangle^{(4)}+\mathcal{O}\left(\epsilon^{6}\right)
$$

for any $n$. 


\section{c. Inner product of FP eigenfunctions including a time derivative}

Since taking a time derivative does not change spatial parity we, similar to the previous case, have for the power series expansion

$$
\left\langle\tilde{\rho}_{n}, \dot{\tilde{\rho}}_{m}\right\rangle=\sum_{l=0}^{\infty} \epsilon^{l}\left\langle\tilde{\rho}_{n}, \dot{\tilde{\rho}}_{m}\right\rangle^{(l)}
$$

that

$$
\left\langle\tilde{\rho}_{n}, \dot{\tilde{\rho}}_{m}\right\rangle^{(l)}=0 \text { if }\left\{\begin{array}{l}
l=0, \\
l>0 \text { odd and } p_{n} p_{m}=1, \\
l>0 \text { even and } p_{n} p_{m}=-1,
\end{array}\right.
$$

where we note that $\dot{\tilde{\rho}}_{m}^{(0)}=0$, since the spectrum of the Laplace operator (inside a unit ball and with time-independent absorbing boundary conditions) is independent of time. In particular we have

$$
\left\langle\tilde{\rho}_{n}, \dot{\tilde{\rho}}_{n}\right\rangle=\epsilon^{2}\left\langle\tilde{\rho}_{n}, \dot{\tilde{\rho}}_{n}\right\rangle^{(2)}+\epsilon^{4}\left\langle\tilde{\rho}_{n}, \dot{\tilde{\rho}}_{n}\right\rangle^{(4)}+\mathcal{O}\left(\epsilon^{6}\right)
$$

for any $n$.

\section{d. Product of the inner product of FP eigenfunctions with a time derivative and the integral over eigenfunctions}

We now consider the power-series expansion of

$$
\left\langle\tilde{\rho}_{m}, \dot{\tilde{\rho}}_{1}\right\rangle \tilde{\mathcal{I}}_{m}=\sum_{k=0}^{\infty} \epsilon^{k}\left(\left\langle\tilde{\rho}_{m}, \dot{\tilde{\rho}}_{1}\right\rangle \tilde{\mathcal{I}}_{m}\right)^{(k)} .
$$

Since $\tilde{\rho}_{1}$ has even parity, $p_{1}=1$, we have according to Eqs. (A43) and (A52) that the expansions in powers of $\epsilon$ of both $\left\langle\tilde{\rho}_{m}, \dot{\tilde{\rho}}_{1}\right\rangle$ and $\tilde{\mathcal{I}}_{m}$ only have nonzero terms at even powers of $\epsilon$ if $p_{m}=1$, and at odd powers of $\epsilon$ if $p_{m}=-1$; therefore, regardless of $p_{m}$ the product $\left\langle\tilde{\rho}_{m}, \dot{\tilde{\rho}}_{1}\right\rangle \tilde{\mathcal{I}}_{m}$ only contains even powers of $\epsilon$, i.e.,

$$
\left(\left\langle\tilde{\rho}_{m}, \dot{\tilde{\rho}}_{1}\right\rangle \tilde{\mathcal{I}}_{m}\right)^{(k)}=0 \text { if } k \text { odd } .
$$

The lowest-order term of the expansion is therefore

$$
\left\langle\tilde{\rho}_{m}, \dot{\tilde{\rho}}_{1}\right\rangle \tilde{\mathcal{I}}_{m}=\epsilon^{2}\left(\left\langle\tilde{\rho}_{m}, \dot{\tilde{\rho}}_{1}\right\rangle \tilde{\mathcal{I}}_{m}\right)^{(2)}+\mathcal{O}\left(\epsilon^{4}\right)
$$

with

$$
\left(\left\langle\tilde{\rho}_{m}, \dot{\tilde{\rho}}_{1}\right\rangle \tilde{\mathcal{I}}_{m}\right)^{(2)}= \begin{cases}\left\langle\tilde{\rho}_{m}, \dot{\tilde{\rho}}_{1}\right\rangle^{(2)} \tilde{\mathcal{I}}_{m}^{(0)} & \text { if } p_{m}=1, \\ \left\langle\tilde{\rho}_{m}, \dot{\tilde{\rho}}_{1}\right\rangle^{(1)} \tilde{\mathcal{I}}_{m}^{(1)} & \text { if } p_{m}=-1 .\end{cases}
$$

\section{APPENDIX B: PERTURBATIVE SOLUTION OF THE FPE}

\section{Perturbative solution of the FPE in terms of the instantaneous spectrum}

We now derive an approximate solution of Eq. (28), which incorporates the coupling between eigenmodes to order $\epsilon^{4}$ (and in the case of a one-dimensional system, $N=1$, to order $\left.\epsilon^{5}\right)$. The following derivation is similar to what in quantum mechanics is called time-dependent perturbation theory $[42,49]$. To render the following calculation easier to read, we rewrite Eq. (28) as

$$
-\dot{\tilde{a}}_{n}=\frac{1}{\epsilon^{2}} \tilde{\Lambda}_{n} \tilde{a}_{n}+\epsilon \sum_{m=1}^{\infty} \tilde{C}_{n m} \tilde{a}_{m},
$$

where we introduce

$$
\begin{gathered}
\tilde{\Lambda}_{n}(\tilde{t}) \equiv \tilde{\lambda}_{n}(\tilde{t})+\left.\epsilon^{2} \frac{\left\langle\tilde{\rho}_{n}, \dot{\tilde{\rho}}_{n}\right\rangle}{\left\langle\tilde{\rho}_{n}, \tilde{\rho}_{n}\right\rangle}\right|_{\tilde{t}}, \\
\left.\tilde{C}_{n m}(\tilde{t}) \equiv\left(1-\delta_{n, m}\right) \frac{1}{\epsilon} \frac{\left\langle\tilde{\rho}_{n}, \dot{\tilde{\rho}}_{m}\right\rangle}{\left\langle\tilde{\rho}_{n}, \tilde{\rho}_{n}\right\rangle}\right|_{\tilde{t}},
\end{gathered}
$$

with $\delta_{n, m}$ the Kronecker delta. From the spectrum calculated in Appendix A 1, it follows that for all $n$ and $m$ we have that $\tilde{\Lambda}_{n}=\mathcal{O}\left(\epsilon^{0}\right)$ and $\tilde{C}_{n m}=\mathcal{O}\left(\epsilon^{0}\right)$, so that the explicit powers of $\epsilon$ on the right-hand side of Eq. (B1) represent the leading-order scaling of each of the terms.

According to Eq. (B1), the dynamics of each mode is for small $\epsilon$ dominated by the adiabatic exponential decay described by the instantaneous decay rate $\tilde{\Lambda}_{n}(\tilde{t}) / \epsilon^{2}$. We now derive an approximate solution to Eq. (B1) which incorporates the leading-order effects of the mode coupling described by the coupling matrix $\tilde{C}_{n m}$.

To separate the adiabatic mode decay and the interaction between modes, we introduce $\tilde{b}_{n}$ via

$$
\tilde{a}_{n}(\tilde{t})=\tilde{b}_{n}(\tilde{t}) \tilde{\mathcal{P}}_{n}^{\mathrm{ad}}\left(\tilde{t}, \tilde{t}_{i}\right)
$$

with the adiabatic propagator $\tilde{\mathcal{P}}_{n}^{\text {ad }}$ for mode $n$ given by

$$
\tilde{\mathcal{P}}_{n}^{\mathrm{ad}}\left(\tilde{t}, \tilde{t}_{i}\right) \equiv \exp \left[-\frac{1}{\epsilon^{2}} \int_{\tilde{t}_{i}}^{\tilde{t}} d \tilde{t}^{\prime} \tilde{\Lambda}_{n}\left(\tilde{t}^{\prime}\right)\right]
$$

Substituting Eq. (B4) into Eq. (B1), we obtain

$$
\dot{\tilde{b}}_{n}(\tilde{t})=-\epsilon \sum_{\substack{m=1 \\ m \neq n}}^{\infty} \tilde{C}_{n m}(\tilde{t}) \tilde{\mathcal{P}}_{m n}^{\mathrm{ad}}\left(\tilde{t}, \tilde{t}_{i}\right) \tilde{b}_{m}(\tilde{t}),
$$

where in the sum bounds we make explicit the fact that $\tilde{C}_{n n}=$ 0 , and where we define

$$
\begin{aligned}
\tilde{\mathcal{P}}_{m n}^{\mathrm{ad}}\left(\tilde{t}, \tilde{t}_{i}\right) & \equiv \tilde{\mathcal{P}}_{m}^{\mathrm{ad}}\left(\tilde{t}, \tilde{t}_{i}\right) / \tilde{\mathcal{P}}_{n}^{\mathrm{ad}}\left(\tilde{t}, \tilde{t}_{i}\right) \\
& =\exp \left[-\frac{1}{\epsilon^{2}} \int_{\tilde{t}_{i}}^{\tilde{t}} d \tilde{t}^{\prime} \Delta \tilde{\Lambda}_{m n}\left(\tilde{t}^{\prime}\right)\right],
\end{aligned}
$$

with $\Delta \tilde{\Lambda}_{m n}(\tilde{t}) \equiv \tilde{\Lambda}_{m}(\tilde{t})-\tilde{\Lambda}_{n}(\tilde{t})$.

Integrating Eq. (B6), we obtain

$$
\tilde{b}_{n}(\tilde{t})=\tilde{b}_{n}\left(\tilde{t}_{i}\right)-\epsilon \sum_{\substack{m=1 \\ m \neq n}}^{\infty} \int_{\tilde{t}_{i}}^{\tilde{t}} d \tilde{t}^{\prime} \tilde{C}_{n m}\left(\tilde{t}^{\prime}\right) \tilde{\mathcal{P}}_{m n}^{\mathrm{ad}}\left(\tilde{t}^{\prime}, \tilde{t}_{i}\right) \tilde{b}_{m}\left(\tilde{t}^{\prime}\right) .
$$

To eliminate $\tilde{b}_{m}\left(\tilde{t}^{\prime}\right)$ in the second term on the right-hand side of Eq. (B9), we reinsert the expression Eq. (B9), similar to the construction of the Dyson series in quantum mechanics [49]. Iterating this procedure, by reinserting Eq. (B9) 
once more in the result, we obtain

$$
\begin{aligned}
& \tilde{b}_{n}(\tilde{t})=\tilde{b}_{n}\left(\tilde{t}_{i}\right)-\epsilon \sum_{\substack{m=1 \\
m \neq n}}^{\infty} \mathcal{M}_{n m}^{(1)}\left(\tilde{t}, \tilde{t}_{i}\right) \tilde{b}_{m}\left(\tilde{t}_{i}\right)+\epsilon^{2} \sum_{\substack{m=1 \\
m \neq n \neq n \\
m \neq n}}^{\infty} \sum_{\substack{k=1 \\
k \neq m}}^{\infty} \mathcal{M}_{n m k}^{(2)}\left(\tilde{t}, \tilde{t}_{i}\right) \tilde{b}_{k}\left(\tilde{t}_{i}\right) \\
&-\epsilon^{3} \sum_{\substack{m=1 \\
m \neq n}}^{\infty} \sum_{\substack{k=1 \\
m \neq m}}^{\infty} \sum_{\substack{l=1 \\
l \neq k}}^{\infty} \int_{\tilde{t}_{i}}^{\tilde{t}} d \tilde{t}^{\prime} \int_{\tilde{t}_{i}}^{\tilde{t}^{\prime}} d \tilde{t}^{\prime \prime} \int_{\tilde{t}_{i}}^{\tilde{t}^{\prime \prime}} d \tilde{t}^{\prime \prime \prime} \tilde{C}_{n m}\left(\tilde{t}^{\prime}\right) \tilde{\mathcal{P}}_{m n}^{\mathrm{ad}}\left(\tilde{t}^{\prime}, \tilde{t}_{i}\right) \tilde{C}_{m k}\left(\tilde{t}^{\prime \prime}\right) \tilde{\mathcal{P}}_{k m}^{\mathrm{ad}}\left(\tilde{t}^{\prime \prime}, \tilde{t}_{i}\right) \tilde{C}_{k l}\left(\tilde{t}^{\prime \prime \prime}\right) \tilde{\mathcal{P}}_{l k}^{\mathrm{ad}}\left(\tilde{t}^{\prime \prime \prime}, \tilde{t}_{i}\right) \tilde{b}_{l}\left(\tilde{t}^{\prime \prime \prime}\right), \\
&
\end{aligned}
$$

where we define

$$
\begin{gathered}
\mathcal{M}_{n m}^{(1)}\left(\tilde{t}, \tilde{t}_{i}\right) \equiv \int_{\tilde{t}_{i}}^{\tilde{t}} d \tilde{t}^{\prime} \tilde{C}_{n m}\left(\tilde{t}^{\prime}\right) \tilde{\mathcal{P}}_{m n}^{\mathrm{ad}}\left(\tilde{t}^{\prime}, \tilde{t}_{i}\right), \\
\mathcal{M}_{n m k}^{(2)}\left(\tilde{t}, \tilde{t}_{i}\right) \equiv \int_{\tilde{t}_{i}}^{\tilde{t}} d \tilde{t}^{\prime} \tilde{C}_{n m}\left(\tilde{t}^{\prime}\right) \tilde{\mathcal{P}}_{m n}^{\mathrm{ad}}\left(\tilde{t}^{\prime}, \tilde{t}_{i}\right) \mathcal{M}_{m k}^{(1)}\left(\tilde{t}^{\prime}, \tilde{t}_{i}\right) .
\end{gathered}
$$

The first term in Eq. (B10) represents the adiabatic decay of the $n$th eigenmode, for which according to Eq. (B4) $\tilde{b}_{n}$ is constant; the remaining terms describe the mode coupling. Intuitively, one might interpret $\mathcal{M}_{n m}^{(1)}$ as describing the direct interaction between two modes $n$ and $m$, and $\mathcal{M}_{n m k}^{(2)}$ as describing the second-order interactions between two modes $n$ and $k$, via an intermediate mode $m$. By successively substituting Eq. (B9) into Eq. (B10), interactions mediated by arbitrary many intermediate modes can be constructed. From the form of Eq. (B9) one might naively expect that to obtain the dynamics of $\tilde{b}_{n}$ to order $\epsilon^{4}$ one needs to substitute Eq. (B9) four times (and therefore discuss interactions mediated by up to two intermediate modes at once). However, since $\tilde{\mathcal{P}}_{n m}^{\mathrm{ad}}$ itself depends on $\epsilon$, the interactions $\mathcal{M}_{n m}^{(1)}$ and $\mathcal{M}_{n m k}^{(2)}$, and their higher-order equivalents, also depend on $\epsilon$; the naive scaling argument that one substitution of Eq. (B9) corresponds to increasing the order in $\epsilon$ by one therefore breaks down. As we discuss now, for the steady-state solution of $\tilde{b}_{n}$ to order $\epsilon^{4}$ (and $\epsilon^{5}$ for a one-dimensional system, $N=1$ ), it is in fact sufficient to discuss the mode-coupling effects mediated by $\mathcal{M}_{n m}^{(1)}$ and $\mathcal{M}_{n m k}^{(2)}$.

\section{Direct interaction between two modes}

To lowest order, the coupling between two modes $n$ and $m$ is given by $\mathcal{M}_{n m}^{(1)}\left(\tilde{t}, \tilde{t}_{i}\right)$ defined in Eq. (B11). To evaluate this matrix element, we distinguish three possible scenarios.

(1) $n<m$, and the eigenvalues $\tilde{\lambda}_{n}$ and $\tilde{\lambda}_{m}$ are not perturbations around the same eigenspace of the Laplace operator, denoted by $n \notin \operatorname{eig}(m)$ (i.e., $\tilde{\lambda}_{n}^{(0)} \neq \tilde{\lambda}_{m}^{(0)}$ ).

(2) $n>m$, and the eigenvalues $\tilde{\lambda}_{n}$ and $\tilde{\lambda}_{m}$ are not perturbations around the same eigenspace of the Laplace operator, denoted by $n \notin \operatorname{eig}(m)$ (i.e., $\left.\tilde{\lambda}_{n}^{(0)} \neq \tilde{\lambda}_{m}^{(0)}\right)$.

(3) $n \neq m$, but the eigenvalues $\tilde{\lambda}_{n}$ and $\tilde{\lambda}_{m}$ are perturbations around the same eigenspace of the Laplace operator, denoted by $n \in \operatorname{eig}(m)$ (i.e., $\left.\tilde{\lambda}_{n}^{(0)}=\tilde{\lambda}_{m}^{(0)}\right)$.

Note that scenario 3 can only occur for dimensions $N \geqslant 2$; in one dimension, $N=1$, the absorbing boundary spectrum of the Laplace operator inside a finite interval is nondegenerate.

\section{a. Direct interactions between modes: Scenario 1}

Since the eigenvalues of the Laplace operator are ordered, for small $\epsilon$ we have $\Delta \tilde{\Lambda}_{m n}(\tilde{t}) \equiv \tilde{\Lambda}_{m}(\tilde{t})-\tilde{\Lambda}_{n}(\tilde{t})>0$, and since $n \notin \operatorname{eig}(m)$ it holds that $\Delta \tilde{\Lambda}_{m n}(\tilde{t})=\mathcal{O}\left(\epsilon^{0}\right)$. Therefore, for small $\epsilon$ the exponential in the definition of $\tilde{\mathcal{P}}_{m n}^{\mathrm{ad}}\left(\tilde{t}, \tilde{t}_{i}\right)$, Eq. (B7), decays on a time scale $\tilde{\tau}_{m n}$ defined by

$$
\int_{\tilde{t}_{i}}^{\tilde{t}_{i}+\tilde{\tau}_{m n}} d \tilde{t}^{\prime} \Delta \tilde{\Lambda}_{m n}\left(\tilde{t}^{\prime}\right)=\epsilon^{2}
$$

so that for small $\epsilon$ we have

$$
\tilde{\tau}_{m n} \approx \epsilon^{2} / \Delta \tilde{\Lambda}_{m n}\left(\tilde{t}_{i}\right)=\mathcal{O}\left(\epsilon^{2}\right) .
$$

Since $\tilde{C}_{n m}=\mathcal{O}\left(\epsilon^{0}\right)$, the integral in Eq. (B11) is in scenario 1 thus dominated by $\tilde{t} \approx \tilde{t}_{i}$. Assuming that $\tilde{C}_{n m}$ does not vary too rapidly on the time scale $\tilde{\tau}_{m n}$, we Taylor expand around $\tilde{t}=\tilde{t}_{i}$ :

$$
\tilde{C}_{n m}(\tilde{t}) \approx \tilde{C}_{n m}\left(\tilde{t}_{i}\right)+\left(\tilde{t}-\tilde{t}_{i}\right) \cdot \dot{\tilde{C}}_{n m}\left(\tilde{t}_{i}\right) .
$$

Furthermore assuming that $\Delta \tilde{\Lambda}_{m n}$ does not vary too much on the decay time scale $\tilde{\tau}_{m n}$, we approximate

$$
\begin{aligned}
\tilde{\mathcal{P}}_{m n}^{\mathrm{ad}}\left(\tilde{t}, \tilde{t}_{i}\right) & =\exp \left[-\frac{1}{\epsilon^{2}} \int_{\tilde{t}_{i}}^{\tilde{t}} d \tilde{t}^{\prime} \Delta \tilde{\Lambda}_{m n}\left(\tilde{t}^{\prime}\right)\right] \\
& \approx \exp \left[-\frac{\tilde{t}-\tilde{t}_{i}}{\epsilon^{2}} \Delta \tilde{\Lambda}_{m n}\left(\tilde{t}_{i}\right)\right] .
\end{aligned}
$$

Physically speaking, with approximations Eqs. (B15) and (B17), we assume that the apparent force (and hence the FP spectrum) inside the tube varies slowly as compared to the relaxation times of the individual modes. Inserting approximations Eqs. (B15) and (B17) into Eq. (B11) the integral is evaluated to

$$
\begin{aligned}
\mathcal{M}_{n m}^{(1)}\left(\tilde{t}, \tilde{t}_{i}\right)= & \frac{\epsilon^{2}}{\Delta \tilde{\Lambda}_{m n}\left(\tilde{t}_{i}\right)}\left\{\tilde{C}_{n m}\left(\tilde{t}_{i}\right)\right. \\
& -\left[\tilde{C}_{n m}\left(\tilde{t}_{i}\right)+\dot{\tilde{C}}_{n m}\left(\tilde{t}_{i}\right) \cdot\left(\tilde{t}-\tilde{t}_{i}\right)\right] \\
& \left.\quad \times \exp \left[-\frac{\tilde{t}-\tilde{t}_{i}}{\epsilon^{2}} \Delta \tilde{\Lambda}_{m n}\left(\tilde{t}_{i}\right)\right]\right\}+\mathcal{O}\left(\epsilon^{5}\right) .
\end{aligned}
$$

For $\tilde{t}-\tilde{t}_{i} \gtrsim \tilde{\tau}_{m n}$, the result Eq. (B18) simplifies to

$$
\mathcal{M}_{n m}^{(1)}\left(\tilde{t}, \tilde{t}_{i}\right)=\epsilon^{2} \frac{\tilde{C}_{n m}\left(\tilde{t}_{i}\right)}{\Delta \tilde{\Lambda}_{m n}\left(\tilde{t}_{i}\right)}+\mathcal{O}\left(\epsilon^{5}\right) .
$$




\section{b. Direct interactions between modes: Scenario 2}

We first note that $\tilde{\mathcal{P}}_{m n}^{\mathrm{ad}}\left(\tilde{t}^{\prime}, \tilde{t}_{i}\right)=\tilde{\mathcal{P}}_{m n}^{\mathrm{ad}}\left(\tilde{t}, \tilde{t}_{i}\right) \tilde{\mathcal{P}}_{n m}^{\mathrm{ad}}\left(\tilde{t}, \tilde{t}^{\prime}\right)$. Substituting this into Eq. (B11), we obtain

$$
\mathcal{M}_{n m}^{(1)}\left(\tilde{t}, \tilde{t}_{i}\right)=\tilde{\mathcal{P}}_{m n}^{\mathrm{ad}}\left(\tilde{t}, \tilde{t}_{i}\right) \int_{\tilde{t}_{i}}^{\tilde{t}} d \tilde{t}^{\prime} \tilde{C}_{n m}\left(\tilde{t}^{\prime}\right) \tilde{\mathcal{P}}_{n m}^{\mathrm{ad}}\left(\tilde{t}, \tilde{t}^{\prime}\right) .
$$

Similar to the discussion of scenario 1 the term $\tilde{\mathcal{P}}_{n m}^{\text {ad }}\left(\tilde{t}, \tilde{t}^{\prime}\right)$ decays exponentially as $\tilde{t}^{\prime}$ is decreased from $\tilde{t}$, with a characteristic decay time scale $\tilde{\tau}_{m n}$ defined by

$$
\int_{\tilde{t}-\tilde{\tau}_{m n}}^{\tilde{t}} d \tilde{t}^{\prime} \Delta \tilde{\Lambda}_{m n}\left(\tilde{t}^{\prime}\right)=\epsilon^{2}
$$

which for small $\epsilon$ is given by

$$
\tilde{\tau}_{m n} \approx \epsilon^{2} / \Delta \tilde{\Lambda}_{m n}(\tilde{t})=\mathcal{O}\left(\epsilon^{2}\right) .
$$

Thus, in scenario 2 the integral in Eq. (B11) is dominated by $\tilde{t}^{\prime} \approx \tilde{t}$. Assuming that $\tilde{C}_{n m}$ does not vary too rapidly on the time scale $\tilde{\tau}_{m n}$, we Taylor expand around $\tilde{t}$ :

$$
\tilde{C}_{n m}\left(\tilde{t}^{\prime}\right) \approx \tilde{C}_{n m}(\tilde{t})+\left(\tilde{t}^{\prime}-\tilde{t}\right) \cdot \dot{\tilde{C}}_{n m}\left(\tilde{t}^{\prime}\right),
$$

where a dot here denotes a derivative with respect to $\tilde{t}$. Furthermore assuming that $\Delta \tilde{\Lambda}_{m n}$ does not vary too much on the time scale $\tilde{\tau}_{m n}$, we approximate

$$
\begin{aligned}
\tilde{\mathcal{P}}_{n m}^{\mathrm{ad}}\left(\tilde{t}, \tilde{t}^{\prime}\right) & =\exp \left[-\frac{1}{\epsilon^{2}} \int_{\tilde{t}^{\prime}}^{\tilde{t}} d \tilde{t}^{\prime} \Delta \tilde{\Lambda}_{n m}\left(\tilde{t}^{\prime}\right)\right] \\
& \approx \exp \left[-\frac{\tilde{t}-\tilde{t}^{\prime}}{\epsilon^{2}} \Delta \tilde{\Lambda}_{n m}(\tilde{t})\right] .
\end{aligned}
$$

Inserting approximations Eqs. (B23) and (B25) into Eq. (B20), in scenario 2 the integral is evaluated to

$$
\begin{aligned}
\mathcal{M}_{n m}^{(1)}\left(\tilde{t}, \tilde{t}_{i}\right)= & \tilde{\mathcal{P}}_{m n}^{\mathrm{ad}}\left(\tilde{t}, \tilde{t}_{i}\right) \frac{\epsilon^{2}}{\Delta \tilde{\Lambda}_{n m}(\tilde{t})} \\
& \times\left\{\tilde{C}_{n m}(\tilde{t})-\left[\tilde{C}_{n m}(\tilde{t})+\dot{\tilde{C}}_{n m}(\tilde{t}) \cdot\left(\tilde{t}-\tilde{t}_{i}\right)\right]\right. \\
& \left.\times \exp \left[-\frac{\tilde{t}-\tilde{t}_{i}}{\epsilon^{2}} \Delta \tilde{\Lambda}_{n m}(\tilde{t})\right]\right\}+\mathcal{O}\left(\epsilon^{5}\right)
\end{aligned}
$$

After an initial transient decay time, i.e., for $\tilde{t}-\tilde{t}_{i} \gtrsim \tilde{\tau}_{n m}$ with $\tilde{\tau}_{m n}$ defined in Eq. (B22), the result Eq. (B26) simplifies to

$$
\mathcal{M}_{n m}^{(1)}\left(\tilde{t}, \tilde{t}_{i}\right)=\epsilon^{2} \tilde{\mathcal{P}}_{m n}^{\mathrm{ad}}\left(\tilde{t}, \tilde{t}_{i}\right) \frac{\tilde{C}_{n m}(\tilde{t})}{\Delta \tilde{\Lambda}_{n m}(\tilde{t})}+\mathcal{O}\left(\epsilon^{5}\right) .
$$

\section{c. Direct interactions between modes: Scenario 3}

According to the perturbative FP spectrum calculated in Appendix A 1, for $n \in \operatorname{eig}(m)$ we have

$$
\Delta \tilde{\Lambda}_{m n}(\tilde{t}) \equiv \tilde{\Lambda}_{m}(\tilde{t})-\tilde{\Lambda}_{n}(\tilde{t})=\mathcal{O}\left(\epsilon^{3}\right)
$$

[see Eqs. (29), (A15), (A20), and (B2), and note that $\left.\left\langle\tilde{\rho}_{n}, \dot{\tilde{\rho}}_{n}\right\rangle=\mathcal{O}(\epsilon)\right]$. It follows that

$$
\tilde{\mathcal{P}}_{m n}^{\mathrm{ad}}\left(\tilde{t}^{\prime}, \tilde{t}_{i}\right)=\exp \left[-\frac{1}{\epsilon^{2}} \int_{\tilde{t}_{i}}^{\tilde{t}} d \tilde{t}^{\prime} \Delta \tilde{\Lambda}_{m n}\left(\tilde{t}^{\prime}\right)\right]=1+\mathcal{O}(\epsilon),
$$

so that to leading order in $\epsilon$ Eq. (B11) becomes

$$
\mathcal{M}_{n m}^{(1)}\left(\tilde{t}, \tilde{t}_{i}\right)=\int_{\tilde{t}_{i}}^{\tilde{t}} d \tilde{t}^{\prime} \tilde{C}_{n m}\left(\tilde{t}^{\prime}\right)+\mathcal{O}(\epsilon) .
$$

\section{d. Direct interactions between modes: Summary}

According to Eqs. (B10), (B19), (B27), and (B30), the leading-order contribution to the coupling between two modes $n$ and $m$ scales with $\epsilon$ as

$$
\epsilon \mathcal{M}_{n m}^{(1)}\left(\tilde{t}, \tilde{t}_{i}\right) \sim \begin{cases}\epsilon^{3}, & n<m \text { and } n \notin \operatorname{eig}(m), \\ \epsilon^{3} \tilde{\mathcal{P}}_{m n}^{\mathrm{ad}}\left(\tilde{t}, \tilde{t}_{i}\right), & n>m \text { and } n \notin \operatorname{eig}(m), \\ \epsilon, & n \in \operatorname{eig}(m),\end{cases}
$$

where $\tilde{\mathcal{P}}_{m n}^{\mathrm{ad}}\left(\tilde{t}, \tilde{t}_{i}\right)$ grows exponentially with an exponent that scales as $1 / \epsilon^{2}$. These scalings are valid after an initial transient time of the order of

$$
\tilde{t}-\tilde{t}_{i} \gtrsim \tilde{\tau}_{m n} \equiv \frac{\epsilon^{2}}{\left|\Delta \tilde{\Lambda}_{m n}\right|},
$$

where we assume that the order of magnitude of $\tilde{\tau}_{m n}$ is independent of the time at which $\Delta \tilde{\Lambda}_{m n}(\tilde{t})$ is evaluated in Eq. (B32), so that we omit the time dependence in $\Delta \tilde{\Lambda}_{m n}$. From the leading-order scalings Eq. (B31) we can infer the largest term in the sum

$$
\epsilon \sum_{\substack{m=1 \\ m \neq n}}^{\infty} \mathcal{M}_{n m}^{(1)}\left(\tilde{t}, \tilde{t}_{i}\right) \tilde{b}_{m}\left(\tilde{t}_{i}\right)
$$

which appears in Eq. (B10). Assuming that all the $\tilde{b}_{m}\left(\tilde{t}_{i}\right)$ are of comparable order of magnitude, which term dominates in Eq. (B33) depends on $n$.

(1) For $n=1$ only scenario 1 is relevant (note that the lowest eigenvalue of the Laplace operator is nondegenerate [50]); the leading-order correction to $\tilde{b}_{n}\left(\tilde{t}_{i}\right)$ is thus at order $\epsilon^{3}$, and all modes $m>1$ contribute to this correction, meaning that all terms in Eq. (B33) are relevant.

(2) For $n>1$ the dominant correction is given by scenario $2, m=1$; this is because the corresponding $\tilde{\mathcal{P}}_{m n}^{\mathrm{ad}}\left(\tilde{t}, \tilde{t}_{i}\right)$ grows fastest, as

$$
\Delta \Lambda_{1 n}=\min _{m<n}\left\{\Delta \Lambda_{m n}\right\},
$$

which follows for small $\epsilon$ from the fact that we perturb around the ordered eigenvalues of the Laplace operator. In particular, note that even though in scenario 3 , where $n \in \operatorname{eig}(m)$, the coupling between modes has a lower-order prefactor (order $\epsilon)$, the fact that in scenario 2 the factor $\tilde{\mathcal{P}}_{m n}^{\text {ad }}\left(\tilde{t}, \tilde{t}_{i}\right)$ grows exponentially (with an exponent that scales as $1 / \epsilon^{2}$ ) makes this the dominant contribution. This means that for $n>1$ the sum Eq. (B33) is dominated by the term $m=1$, i.e.,

$$
\epsilon \sum_{\substack{m=1 \\ m \neq n}}^{\infty} \mathcal{M}_{n m}^{(1)}\left(\tilde{t}, \tilde{t}_{i}\right) \tilde{b}_{m}\left(\tilde{t}_{i}\right) \approx \epsilon \mathcal{M}_{n 1}^{(1)}\left(\tilde{t}, \tilde{t}_{i}\right) \tilde{b}_{1}\left(\tilde{t}_{i}\right),
$$

which is expected to hold after a time $\tilde{\tau}_{1 n}$ as defined in Eq. (B32). 
Intuitively, these results tell us that (i) the dominant correction to the adiabatic decay of the lowest mode $n=1$ is due to its interaction with the modes $m>1$ during the initial relaxation of the initial conditions [note that $\tilde{C}_{n m}$ and $\Delta \tilde{\Lambda}_{m n}$ in Eq. (B19) are evaluated at $\left.\tilde{t}_{i}\right]$ and (ii) the dominant correction to the adiabatic decay of any mode $n>1$ is due to instantaneous excitation by the lowest mode $m=1$ [note that $\tilde{C}_{n m}$ and $\Delta \tilde{\Lambda}_{m n}$ in Eq. (B27) are evaluated at $\left.\tilde{t}\right]$, which after an initial relaxation is expected to be the dominant mode.

\section{Higher-order coupling between modes}

From Eq. (B31) and the subsequent discussion we see that after the initial relaxation of the system the interaction between two modes $n \neq m$ leads to corrections of order $\epsilon^{3}$ if $n \notin \operatorname{eig}(m)$ (with an exponentially growing factor if $n>m$ ), and of order $\epsilon$ if $n \in \operatorname{eig}(m)$. To calculate the leading-order corrections to $\tilde{b}_{n}$ up to order $\epsilon^{4}$ in the steady-state limit, we therefore only need to take into account two scenarios for the three-mode coupling described by Eq. (B12).

(1) $k>1, m \in \operatorname{eig}(k), n=1$. In this scenario, a mode $k>$ 1 couples to a mode $m \neq k$ from the same Laplace eigenspace ( $\rightarrow$ interaction of order $\epsilon$ ), which then couples to the lowest mode $n=1$ ( $\rightarrow$ interaction of order $\left.\epsilon^{3}\right)$.

(2) $k=1, m>1, n \in \operatorname{eig}(m)$. In this scenario, the lowest mode $k=1$ excites a mode $m>1\left(\rightarrow\right.$ interaction of order $\epsilon^{3}$, with an exponentially growing prefactor), which then couples to a mode $n \neq m$ from the same Laplace eigenspace $(\rightarrow$ interaction of order $\epsilon$ ).

Note that these cases are only relevant for dimensions $N \geqslant$ 2; since for $N=1$ the spectrum of the Laplace operator is not degenerate, higher-order couplings between modes always scale as $\epsilon^{6}$ for a one-dimensional system.

\section{a. Higher-order coupling between modes: Scenario 1}

Since $1=n<m$ the factor $\tilde{\mathcal{P}}_{m n}^{\mathrm{ad}}\left(\tilde{t}^{\prime}, \tilde{t}_{i}\right)$ decays exponentially as a function of $\tilde{t}^{\prime}$, so that the $\tilde{t}^{\prime}$ integral in Eq. (B12) is dominated by $\tilde{t}^{\prime} \approx \tilde{t}_{i}$. We therefore approximate

$$
\tilde{\mathcal{P}}_{m n}^{\mathrm{ad}}\left(\tilde{t}^{\prime}, \tilde{t}_{i}\right) \approx \exp \left[-\frac{\tilde{t}^{\prime}-\tilde{t}_{i}}{\epsilon^{2}} \Delta \tilde{\Lambda}_{m n}\left(\tilde{t}_{i}\right)\right],
$$

and furthermore Taylor expand

$$
\begin{gathered}
\tilde{C}_{n m}\left(\tilde{t}^{\prime}\right) \approx \tilde{C}_{n m}\left(\tilde{t}_{i}\right)+\left(\tilde{t}-\tilde{t}^{\prime}\right) \cdot \dot{\tilde{C}}_{n m}\left(\tilde{t}_{i}\right), \\
\mathcal{M}_{m k}^{(1)}\left(\tilde{t}^{\prime}, \tilde{t}_{i}\right) \approx\left(\tilde{t}^{\prime}-\tilde{t}_{i}\right) \cdot \tilde{C}_{m k}\left(\tilde{t}_{i}\right)+\frac{1}{2}\left(\tilde{t}^{\prime}-\tilde{t}_{i}\right)^{2} \cdot \dot{\tilde{C}}_{m k}\left(\tilde{t}_{i}\right)+\mathcal{O}(\epsilon),
\end{gathered}
$$

where at the last equality sign we use that for $m \in \operatorname{eig}(k)$ we have $\tilde{\mathcal{P}}_{k m}^{\text {ad }}\left(\tilde{t}^{\prime \prime}, \tilde{t}_{i}\right)=1+\mathcal{O}(\epsilon)$ [see Eq. (B29)]. Substituting Eqs. (B36)-(B38) into Eq. (B12), the $\tilde{t}^{\prime}$ integral is evaluated using integration by parts to yield

$$
\begin{aligned}
\mathcal{M}_{n m k}^{(2)}\left(\tilde{t}, \tilde{t}_{i}\right)= & -\epsilon^{2} \frac{\tilde{t}-\tilde{t}_{i}}{\Delta \tilde{\Lambda}_{m n}\left(\tilde{t}_{i}\right)}\left[\tilde{C}_{n m}\left(\tilde{t}_{i}\right)+\left(\tilde{t}-\tilde{t}_{i}\right) \cdot \dot{\tilde{C}}_{n m}\left(\tilde{t}_{i}\right)\right] \\
& \times\left[\tilde{C}_{m k}\left(\tilde{t}_{i}\right)+\frac{1}{2}\left(\tilde{t}-\tilde{t}_{i}\right) \cdot \dot{\tilde{C}}_{m k}\left(\tilde{t}_{i}\right)\right] \\
& \times \exp \left[-\frac{\tilde{t}-\tilde{t}_{i}}{\epsilon^{2}} \Delta \tilde{\Lambda}_{m n}\left(\tilde{t}_{i}\right)\right]+\mathcal{O}\left(\epsilon^{3}\right),
\end{aligned}
$$

which vanishes (to order $\epsilon^{2}$ ) as $\tilde{t}-\tilde{t}_{i} \gtrsim \tilde{\tau}_{m 1}$ (recall that in the current scenario $n=1$ ), with $\tilde{\tau}_{m 1}$ defined in Eq. (B32).

\section{b. Higher-order coupling between modes: Scenario 2}

Exchanging the two integrals that are present in Eq. (B12) after substituting Eq. (B11), we obtain

$$
\begin{aligned}
\mathcal{M}_{n m k}^{(2)}\left(\tilde{t}, \tilde{t}_{i}\right)= & \tilde{\mathcal{P}}_{k m}^{\mathrm{ad}}\left(\tilde{t}, \tilde{t}_{i}\right) \int_{\tilde{t}_{i}}^{\tilde{t}} d \tilde{t}^{\prime \prime} \tilde{C}_{m k}\left(\tilde{t}^{\prime \prime}\right) \tilde{\mathcal{P}}_{m k}^{\mathrm{ad}}\left(\tilde{t}, \tilde{t}^{\prime \prime}\right) \\
& \times \int_{\tilde{t}^{\prime \prime}}^{\tilde{t}} d \tilde{t}^{\prime} \tilde{C}_{n m}\left(\tilde{t}^{\prime}\right) \tilde{\mathcal{P}}_{m n}^{\mathrm{ad}}\left(\tilde{t}^{\prime}, \tilde{t}_{i}\right),
\end{aligned}
$$

where we use $\tilde{\mathcal{P}}_{k m}^{\mathrm{ad}}\left(\tilde{t}^{\prime \prime}, \tilde{t}_{i}\right)=\tilde{\mathcal{P}}_{k m}^{\mathrm{ad}}\left(\tilde{t}, \tilde{t}_{i}\right) \tilde{\mathcal{P}}_{m k}^{\mathrm{ad}}\left(\tilde{t}, \tilde{t}^{\prime \prime}\right)$. Since $1=$ $k<m$, the factor $\tilde{\mathcal{P}}_{m k}^{\text {ad }}\left(\tilde{t}, \tilde{t}^{\prime \prime}\right)$ decays exponentially as $\tilde{t}^{\prime \prime}$ is decreased from $\tilde{t}$, so that the $\tilde{t}^{\prime \prime}$ integral is dominated by $\tilde{t}^{\prime \prime} \approx \tilde{t}$. Similar to scenario 1 , we therefore approximate

$$
\begin{aligned}
& \tilde{\mathcal{P}}_{m k}^{\mathrm{ad}}\left(\tilde{t}, \tilde{t}^{\prime \prime}\right) \approx \exp \left[-\frac{\tilde{t}-\tilde{t}^{\prime \prime}}{\epsilon^{2}} \Delta \tilde{\Lambda}_{m k}(\tilde{t})\right] \\
& \tilde{C}_{m k}\left(\tilde{t}^{\prime \prime}\right) \approx \tilde{C}_{m k}(\tilde{t})+\left(\tilde{t}^{\prime \prime}-\tilde{t}\right) \cdot \dot{\tilde{C}}_{n m}(\tilde{t}), \\
& \int_{\tilde{t}^{\prime \prime}}^{\tilde{t}} d \tilde{t}^{\prime} \tilde{C}_{n m}\left(\tilde{t}^{\prime}\right) \tilde{\mathcal{P}}_{m n}^{\mathrm{ad}}\left(\tilde{t}^{\prime}, \tilde{t}_{i}\right) \\
& \approx\left(\tilde{t}-\tilde{t}^{\prime \prime}\right) \cdot \tilde{C}_{n m}(\tilde{t})+\frac{\left(\tilde{t}-\tilde{t}^{\prime \prime}\right)^{2}}{2} \dot{\tilde{C}}_{n m}(\tilde{t})+\mathcal{O}(\epsilon),
\end{aligned}
$$

where at the last equality sign we use that for $m \in \operatorname{eig}(n)$ we have $\tilde{\mathcal{P}}_{m n}^{\mathrm{ad}}\left(\tilde{t}^{\prime}, \tilde{t}_{i}\right)=1+\mathcal{O}(\epsilon)$ [see Eq. (B29)]. Substituting Eqs. (B41)-(B43) into Eq. (B40), the $\tilde{t}^{\prime \prime}$ integral is then evaluated using integration by parts to yield

$$
\begin{aligned}
\mathcal{M}_{n m k}^{(2)}\left(\tilde{t}, \tilde{t}_{i}\right)= & -\tilde{\mathcal{P}}_{k m}^{\mathrm{ad}}\left(\tilde{t}, \tilde{t}_{i}\right) \epsilon^{2} \frac{\tilde{t}-\tilde{t}_{i}}{\Delta \tilde{\Lambda}_{m k}(\tilde{t})} \\
& \times\left[\tilde{C}_{m k}(\tilde{t})-\left(\tilde{t}-\tilde{t}_{i}\right) \cdot \dot{\tilde{C}}_{m k}(\tilde{t})\right] \\
& \times\left[\tilde{C}_{n m}(\tilde{t})-\frac{1}{2}\left(\tilde{t}-\tilde{t}_{i}\right) \cdot \dot{\tilde{C}}_{n m}(\tilde{t})\right] \\
& \times \exp \left[-\frac{\tilde{t}-\tilde{t}_{i}}{\epsilon^{2}} \Delta \tilde{\Lambda}_{m k}(\tilde{t})\right]+\mathcal{O}\left(\epsilon^{3}\right) .
\end{aligned}
$$

Comparing this result to Eq. (B27), we see that after an initial transient time, i.e., for $\tilde{t}-\tilde{t}_{i} \gtrsim \tilde{\tau}_{21}=\max _{m>1}\left\{\tilde{\tau}_{m 1}\right\}$ (recall that in the current scenario $k=1$ ), with $\tilde{\tau}_{m 1}$ defined in Eq. (B32), the contribution to the amplitude $\tilde{b}_{n}$ from Eq. (B44) is exponentially smaller than the contribution from Eq. (B27); thus the contribution from Eq. (B44) can be neglected as $\tilde{t}-\tilde{t}_{i} \gtrsim$ $\tilde{\tau}_{21}$.

\section{Final result for the approximate FP solution}

Substituting the results Eqs. (B19), (B27), (B30), (B39), and (B44) into Eq. (B10), we find that the $\tilde{b}_{n}$ are to exponentially leading order given by

$$
\begin{gathered}
\tilde{b}_{1}(\tilde{t}) \approx \tilde{b}_{1}\left(\tilde{t}_{i}\right)-\epsilon^{3} \sum_{m=2}^{\infty} \frac{\tilde{C}_{1 m}\left(\tilde{t}_{i}\right)}{\Delta \tilde{\Lambda}_{m 1}\left(\tilde{t}_{i}\right)} \tilde{b}_{m}\left(\tilde{t}_{i}\right)+\mathcal{O}\left(\epsilon^{k}\right), \\
\tilde{b}_{n}(\tilde{t}) \approx-\tilde{\mathcal{P}}_{1 n}^{\mathrm{ad}}\left(\tilde{t}, \tilde{t}_{i}\right) \epsilon^{3} \frac{\tilde{C}_{n 1}(\tilde{t})}{\Delta \tilde{\Lambda}_{n 1}(\tilde{t})} \tilde{b}_{1}\left(\tilde{t}_{i}\right)+\mathcal{O}\left(\epsilon^{k}\right),
\end{gathered}
$$


where $n>1$ in Eq. (B46); $k=6$ for a one-dimensional system, $N=1$; and $k=5$ for $N \geqslant 2$. These approximate expressions are valid after an initial transient decay time

$$
\tilde{t}-\tilde{t}_{i} \gtrsim \tilde{\tau}_{\text {rel }} \equiv \tilde{\tau}_{21}=\max _{m>1}\left\{\tilde{\tau}_{m 1}\right\},
$$

with $\tilde{\tau}_{m 1}$ defined in Eq. (B32). Substituting these results for $\tilde{b}_{n}$ into Eq. (B4), the coefficients $\tilde{a}_{n}$ of the eigenfunction expansion of the solution of the FPE are finally given by

$$
\begin{aligned}
\tilde{a}_{1}(\tilde{t}) \approx & \tilde{\mathcal{P}}_{1}^{\mathrm{ad}}\left(\tilde{t}, \tilde{t}_{i}\right)\left[\tilde{a}_{1}\left(\tilde{t}_{i}\right)\right. \\
& \left.-\epsilon^{3} \sum_{m=2}^{\infty} \frac{\tilde{C}_{1 m}\left(\tilde{t}_{i}\right)}{\Delta \tilde{\Lambda}_{m 1}\left(\tilde{t}_{i}\right)} \tilde{a}_{m}\left(\tilde{t}_{i}\right)+\mathcal{O}\left(\epsilon^{k}\right)\right], \\
\tilde{a}_{n}(\tilde{t}) \approx & \tilde{\mathcal{P}}_{1}^{\mathrm{ad}}\left(\tilde{t}, \tilde{t}_{i}\right)\left[-\epsilon^{3} \frac{\tilde{C}_{n 1}(\tilde{t})}{\Delta \tilde{\Lambda}_{n 1}(\tilde{t})} \tilde{b}_{1}\left(\tilde{t}_{i}\right)+\mathcal{O}\left(\epsilon^{k}\right)\right] \\
= & -\epsilon^{3} \frac{\tilde{C}_{n 1}(\tilde{t})}{\Delta \tilde{\Lambda}_{n 1}(\tilde{t})} \tilde{a}_{1}(\tilde{t})+\mathcal{O}\left(\epsilon^{k}\right),
\end{aligned}
$$

where $n>1$, and for a one-dimensional system, $N=1$, we have $k=6$, while for $N \geqslant 2$ we have $k=5$; to obtain Eqs. (B48)-(B50) we furthermore use that $\tilde{a}_{n}\left(\tilde{t}_{i}\right)=\tilde{b}_{n}\left(\tilde{t}_{i}\right)$, and at Eq. (B50) we use Eq. (B48). The expressions Eqs. (B48) and (B49) hold after the initial transient decay time $\tilde{\tau}_{\text {rel }}$ defined in Eq. (B47), and neglect both terms of the order $\mathcal{O}\left(\epsilon^{k}\right)$, as well as terms exponentially small as compared to the leadingorder contributions.

\section{APPENDIX C: EXPLICIT RESULTS FOR ONE-DIMENSIONAL SYSTEMS}

In the present section, we consider our theory for a onedimensional system, $N=1$.

\section{Spectrum of the FPE}

We now derive explicit expressions for the perturbative spectrum of the FPE, following the strategy from Appendix A 1. In particular we show that at order $k$ the perturbative contribution to the eigenfunction is given by

$$
\begin{aligned}
\tilde{\rho}_{n}^{(k)}(\tilde{x}, \tilde{t})= & \tilde{Q}_{n, \mathrm{~s}}^{(k)}(\tilde{x}, \tilde{t}) \sin \left[n \frac{\pi}{2}(\tilde{x}+1)\right] \\
& +\tilde{Q}_{n, \mathrm{c}}^{(k)}(\tilde{x}, \tilde{t}) \cos \left[n \frac{\pi}{2}(\tilde{x}+1)\right],
\end{aligned}
$$

where $\tilde{Q}_{n, \mathrm{~s}}^{(k)}(\tilde{x}, \tilde{t})$ and $\tilde{Q}_{n, \mathrm{c}}^{(k)}(\tilde{x}, \tilde{t})$ are polynomials in $\tilde{x}$ of order $\leqslant k$.

For $N=1$, the Taylor expansion of the force, Eqs. (A1), becomes

$$
\tilde{F}_{\text {app }}(\tilde{x}, \tilde{t})=-\sum_{k=1}^{\infty} \epsilon^{k-1} k \tilde{E}_{k}(\tilde{t}) \tilde{x}^{k-1},
$$

with

$$
\tilde{E}_{k}(\tilde{t}) \equiv-\left.\frac{1}{k !} L^{k} \beta \frac{\partial^{k-1} F}{\partial x^{k-1}}\right|_{(\varphi(t), t)}+\delta_{k, 1} \dot{\tilde{\varphi}}(\tilde{t}),
$$

where $(x, t)$ and $(\tilde{x}, \tilde{t})$ are related via Eq. (14). With this, the equation for the $n$th eigenvalue/eigenfunction pair at order $\epsilon^{k}$,
Eq. (A8), becomes

$$
\begin{aligned}
\partial_{\tilde{x}}^{2} \tilde{\rho}_{n}^{(k)}+\tilde{\lambda}_{n}^{(0)} \tilde{\rho}_{n}^{(k)}= & -\sum_{l=1}^{k} \tilde{\lambda}_{n}^{(l)} \tilde{\rho}_{n}^{(k-l)} \\
& -\sum_{l=1}^{k} l \tilde{E}_{l} \partial_{\tilde{x}}\left(\tilde{x}^{l-1} \tilde{\rho}_{n}^{(k-l)}\right),
\end{aligned}
$$

where we use the convention that for $k=0$ the sums on the right-hand side are zero, and each $\tilde{\rho}_{n}^{(k)}$ fulfills the boundary conditions

$$
\tilde{\rho}_{n}^{(k)}(\tilde{x}=-1, \tilde{t})=\tilde{\rho}_{n}^{(k)}(\tilde{x}=1, \tilde{t})=0
$$

[see Eq. (A9)]. The normalization condition at order $k$ is given by Eqs. (A10) and (A11), where we note that

$$
\tilde{\rho}_{\mathrm{SS}}^{-1}(\tilde{x}, \tilde{t})=\exp \left[\sum_{k=1}^{\infty} \epsilon^{k} \tilde{E}_{k}(\tilde{t}) \tilde{x}^{k}\right] .
$$

For $N=1$ the equation for the $\tilde{\lambda}_{n}^{(k)}$, Eq. (A12), becomes

$$
\begin{aligned}
\tilde{\lambda}_{n}^{(k)}= & -\sum_{l=1}^{k-1} \tilde{\lambda}_{n}^{(l)} \int_{-1}^{1} d \tilde{x} \tilde{\rho}_{n}^{(0)} \tilde{\rho}_{n}^{(k-l)} \\
& -\sum_{l=1}^{k} l \int_{\tilde{B}} d \tilde{x} \tilde{\rho}_{n}^{(0)} \tilde{E}_{l} \partial_{\tilde{x}}\left(\tilde{x}^{l-1} \tilde{\rho}_{n}^{(k-l)}\right) .
\end{aligned}
$$

We now show how Eqs. (C4), (C5), and (C7), can be solved recursively with increasing $k$, and that at order $k$ the solution for $\tilde{\rho}_{n}^{(k)}$ is of the form Eq. (C1).

At order $k=0$, the right-hand side of Eq. (C4) vanishes and we obtain

$$
\begin{gathered}
\tilde{\lambda}_{n}^{(0)}=\left(\frac{n \pi}{2}\right)^{2}, \\
\tilde{\rho}_{n}^{(0)}(\tilde{x})=\sin \left[n \frac{\pi}{2}(\tilde{x}+1)\right],
\end{gathered}
$$

which is simply the spectrum for free diffusion in a domain $\tilde{x} \in[-1,1]$ with absorbing boundary conditions. Note that Eq. (C9) fulfills the normalization condition Eq. (A10).

Assuming the spectrum is known to order $k-1$ and is of the form Eq. (C1), we now derive expressions for $\tilde{\lambda}_{n}^{(k)}$ and $\tilde{\rho}_{n}^{(k)}$. The correction at order $k$ to the eigenvalue, $\tilde{\lambda}_{n}^{(k)}$, is obtained directly from Eq. (C7) by evaluating the right-hand side. According to Eq. (C1) for $n<k$, the integrands on the right-hand side of Eq. (C7) are sums over trigonometric functions multiplied by powers of $\tilde{x}$; evaluating these integrals in practice is thus straightforward. We now turn to calculating $\tilde{\rho}_{n}^{(k)}$, which according to Eq. (C4) obeys an inhomogeneous (undamped) harmonic oscillator equation of motion. The solution thus has the general form

$$
\tilde{\rho}_{n}^{(k)}(\tilde{x})=\tilde{\rho}_{n, \text { hom }}^{(k)}(\tilde{x})+\tilde{\rho}_{n, \text { inhom }}^{(k)}(\tilde{x}),
$$

where

$$
\tilde{\rho}_{n, \text { hom }}^{(k)}(\tilde{x})=\tilde{A}_{n}^{(k)} \sin \left[n \frac{\pi}{2}(\tilde{x}+1)\right]+\tilde{B}_{n}^{(k)} \cos \left[n \frac{\pi}{2}(\tilde{x}+1)\right]
$$

is the homogeneous harmonic oscillator solution (the coefficients $\tilde{A}_{n}^{(k)}$ and $\tilde{B}_{n}^{(k)}$ will be determined below), and $\tilde{\rho}_{n, \text { inhom }}^{(k)}$ 
is an inhomogeneous solution of Eq. (C4). To obtain an inhomogeneous solution we note that according to Eq. (C1) the right-hand side of Eq. (C4) is a sum over the trigonometric functions $\sin$ and cos, multiplied by powers $\tilde{x}^{l}, l \leqslant k-1$. As we show in Appendix C 2, an inhomogeneous solution $\tilde{\rho}_{n \text {,inhom }}^{(k)}$ to Eq. (C4) is then again given by a sum over trigonometric functions $\sin$ and cos, multiplied by powers $\tilde{x}^{l}$ with $l \leqslant k$. Thus, Eq. (C10) is again of the form Eq. (C4).

After an inhomogeneous solution at a given order $k$ has been calculated using the formulas from Appendix $C 2$, the coefficient $\tilde{B}_{n}^{(k)}$ in Eq. (C10) is fixed by the boundary conditions Eq. (C5). The remaining coefficient $\tilde{A}_{n}^{(k)}$ is determined by the normalization condition Eq. (A11).
Using this algorithm, the spectrum can be calculated to arbitrary order in $\epsilon^{k}$. While according to Eq. (A15) $\tilde{\lambda}_{n}^{(k)}=0$ for $k$ odd, for $k \leqslant 5$ the eigenvalues for even $k$ follow as

$$
\begin{gathered}
\tilde{\lambda}_{n}^{(0)}=\left(\frac{n \pi}{2}\right)^{2}, \quad \tilde{\lambda}_{n}^{(2)}=\left(\frac{\tilde{E}_{1}}{2}\right)^{2}-\tilde{E}_{2}, \\
\tilde{\lambda}_{n}^{(4)}=\frac{1}{6(n \pi)^{2}}\left(3 E_{1} E_{3}+2 E_{2}^{2}-12 E_{4}\right)\left[(n \pi)^{2}-6\right] .
\end{gathered}
$$

The corresponding eigenfunctions for $l \leqslant 5$ are of the form Eq. (C4), with polynomials

$$
\begin{gathered}
\tilde{Q}_{n, \mathrm{~s}}^{(0)}(\tilde{x})=1, \quad \tilde{Q}_{n, \mathrm{c}}^{(0)}(\tilde{x})=0, \\
\tilde{Q}_{n, \mathrm{~s}}^{(1)}(\tilde{x})=-\frac{\tilde{E}_{1}}{2} \tilde{x}, \quad \tilde{Q}_{n, \mathrm{c}}^{(1)}(\tilde{x})=0, \\
\tilde{Q}_{n, \mathrm{~s}}^{(2)}(\tilde{x})=\frac{\tilde{x}^{2}}{8}\left(\tilde{E}_{1}^{2}-4 \tilde{E}_{2}\right), \quad \tilde{Q}_{n, \mathrm{c}}^{(2)}(\tilde{x})=0, \\
\tilde{Q}_{n, \mathrm{~s}}^{(3)}(\tilde{x})=\frac{\tilde{x}}{48(n \pi)^{2}}\left\{-\tilde{E}_{1}^{3}(n \pi \tilde{x})^{2}+12 \tilde{E}_{1} \tilde{E}_{2}\left[(n \pi \tilde{x})^{2}+4\right]-24 \tilde{E}_{3}\left[(n \pi \tilde{x})^{2}+6\right]\right\}, \\
\tilde{Q}_{n, \mathrm{c}}^{(3)}(\tilde{x})=-\frac{1}{2 n \pi}\left(\tilde{x}^{2}-1\right)\left(\tilde{E}_{1} \tilde{E}_{2}-3 \tilde{E}_{3}\right), \\
\tilde{Q}_{n, \mathrm{~s}}^{(4)}(\tilde{x})=\frac{1}{384(n \pi)^{4}}\left\{\tilde{E}_{1}^{4}(n \pi \tilde{x})^{4}-24 \tilde{E}_{1}^{2} \tilde{E}_{2}(n \pi \tilde{x})^{2}\left[(n \pi \tilde{x})^{2}+8\right]+96 \tilde{E}_{3} \tilde{E}_{1}\left[(n \pi \tilde{x})^{4}+2(n \pi)^{2}\left(6 \tilde{x}^{2}-1\right)+24\right]\right. \\
\left.+16 \tilde{E}_{2}^{2}\left[3(n \pi \tilde{x})^{4}+8(n \pi)^{2}\left(3 \tilde{x}^{2}-1\right)+96\right]+192 \tilde{E}_{4}\left[-(n \pi \tilde{x})^{4}+4(n \pi)^{2}\left(-3 \tilde{x}^{2}+1\right)-48\right]\right\} \\
\tilde{Q}_{n, \mathrm{~s}}^{(5)}(\tilde{x})=\frac{1}{3840(n \pi)=}(\tilde{x})=\frac{1}{12 n \pi} \tilde{x}^{4}\left(\tilde{x}^{2}-1\right)\left(3 \tilde{E}_{1}^{2} \tilde{E}_{2}-15 \tilde{E}_{1} \tilde{E}_{3}-4 \tilde{E}_{2}^{2}+24 \tilde{E}_{4}\right), \\
+240 \tilde{E}_{1}^{2} \tilde{E}_{3}\left[-(n \pi \tilde{x})^{4}+40 \tilde{E}_{1}^{3} \tilde{E}_{2}(n \pi \tilde{x})^{2}\left[(n \pi \tilde{x})^{2}+12\right]-80 \tilde{E}_{1} \tilde{E}_{2}^{2}\left[3(n \pi \tilde{x})^{4}+8(n \pi)^{2}\left(6 \tilde{x}^{2}-1\right)+96\right]\right. \\
+960 \tilde{E}_{2} \tilde{E}_{3}\left[(n \pi \tilde{x})^{4}+18(n \pi)^{2}\left(-9 \tilde{x}^{2}+2\right)-48\right]+960 \tilde{E}_{1} \tilde{E}_{4}(n \pi)^{2}\left[(n \pi)^{2} \tilde{x}^{4}+20 \tilde{x}^{2}-4\right] \\
\frac{1}{96(n \pi)^{3}}\left(\tilde{x}^{2}-1\right)\left\{-6 \tilde{E}_{1}^{3} \tilde{E}_{2}(n \pi \tilde{x})^{2}+40 \tilde{E}_{1} \tilde{E}_{2}^{2}(n \pi \tilde{x})^{2}+42 \tilde{E}_{1}^{2} \tilde{E}_{3}(n \pi \tilde{x})^{2}\right. \\
\left.-48 \tilde{E}_{1} \tilde{E}_{4}\left[(n \pi)^{2}\left(3 \tilde{x}^{2}+1\right)-12\right]-24 \tilde{E}_{2} \tilde{E}_{3}\left[3(n \pi)^{2}\left(2 \tilde{x}^{2}+1\right)-36\right]+240 \tilde{E}_{5}\left[(n \pi)^{2}\left(\tilde{x}^{2}+1\right)-12\right]\right\},
\end{gathered}
$$

where we suppress the time dependence via the $\tilde{E}_{l}$, which are defined in Eq. (C3).

With Eqs. (29), (A15), (C1), (C3), and (C12)-(C22), we have explicit expressions for the spectrum of the FP operator Eq. (21) up to order $\epsilon^{6}$ (eigenvalues) and $\epsilon^{5}$ (eigenfunctions) for the case of a one-dimensional system, $N=1$.

\section{Inhomogeneous solution for the harmonic oscillator}

In the present section we explain how to obtain an inhomogeneous solution to Eq. (C4). Since Eq. (C4) is linear, we can consider the inhomogeneous solution for each term on the right-hand side separately, and subsequently add them. According to Eqs. (C1) and (C9), for each term on the righthand side of Eq. (C4) we have to solve an equation of either of the two forms

$$
\begin{aligned}
& \partial_{\tilde{x}}^{2} \tilde{\rho}_{n, \text { inhom }}+\left(\frac{n \pi}{2}\right)^{2} \tilde{\rho}_{n, \text { inhom }}=T \tilde{x}^{l} \sin \left[n \frac{\pi}{2}(\tilde{x}+1)\right] \\
& \partial_{\tilde{x}}^{2} \tilde{\rho}_{n, \text { inhom }}+\left(\frac{n \pi}{2}\right)^{2} \tilde{\rho}_{n, \text { inhom }}=T \tilde{x}^{l} \cos \left[n \frac{\pi}{2}(\tilde{x}+1)\right]
\end{aligned}
$$

with $T \in \mathbb{R}, l \in\{0,1,2, .$.$\} and n \in\{1,2, \ldots\}$. Direct substitution shows that while a solution to Eq. (C23) is given by

$$
\begin{aligned}
\tilde{\rho}_{n, \text { inhom }}(\tilde{x})= & T \sum_{m=1}^{l+1} \frac{l !}{m !} \frac{(n \pi \tilde{x})^{m}}{(n \pi)^{l+2}} \\
& \times \sin \left[n \frac{\pi}{2}(\tilde{x}+1)+\frac{\pi}{2}(l-m)\right]
\end{aligned}
$$


an inhomogeneous solution to Eq. (C24) is given by

$$
\begin{aligned}
\tilde{\rho}_{n, \text { inhom }}(\tilde{x})= & T \sum_{m=1}^{l+1} \frac{l !}{m !} \frac{(n \pi \tilde{x})^{m}}{(n \pi)^{l+2}} \\
& \times \cos \left[n \frac{\pi}{2}(\tilde{x}+1)+\frac{\pi}{2}(l-m)\right] .
\end{aligned}
$$

Note that the shifts $(l-m) \pi / 2$ with $l-m \in \mathbb{Z}$ in Eqs. (C25) and (C26) simply alternate between cos and sin (with possible sign changes), as follows directly from the trigonometric identities $\sin (\theta \pm \pi / 2)= \pm \cos \theta$ and $\cos (\theta \pm \pi / 2)=\mp \sin \theta$. Thus, each term in the solutions Eqs. (C25) and (C26) is again of the form of the right-hand side of Eqs. (C23) and (C24). In particular, if we start with a power $\tilde{x}^{l}$ in either Eq. (C23) or Eq. (C24), the highest power in $\tilde{x}$ that appears in the corresponding inhomogeneous solution Eq. (C25) is $l+1$. From this and Eq. (C9) it follows that the order of the polynomials $\tilde{Q}_{n, \mathrm{~s}}^{(k)}(\tilde{x})$ and $\tilde{Q}_{n, \mathrm{c}}^{(k)}(\tilde{x})$ is always less than or equal to $k$.

\section{Normalized probability density inside the tube to order $\epsilon^{5}$}

In the present section we give the explicit expansion of the normalized probability density Eqs. (39) as a power series in $\epsilon$ up to order $\epsilon^{5}$. The power series is based on the un-normalized density Eq. (36) and has the form Eq. (53), reprinted here for convenience:

$$
\begin{aligned}
\tilde{P}_{\epsilon}^{n, \varphi}(\tilde{x}, \tilde{t})= & \sum_{k=0}^{4} \epsilon^{k}\left\{\tilde{N}_{\mathrm{s}}^{(k)} \sin \left[\frac{\pi}{2}(\tilde{x}+1)\right]\right. \\
& \left.+\tilde{N}_{\mathrm{c}}^{(k)} \cos \left[\frac{\pi}{2}(\tilde{x}+1)\right]\right\}+\mathcal{O}\left(\epsilon^{6}\right) .
\end{aligned}
$$

Substituting the perturbative FP spectrum calculated in Appendix C 1 into the propagator Eq. (36), the infinite sums that appear can be evaluated explicitly. Using the result to evaluate the normalized probability density Eq. (39), an explicit perturbative expression for $\tilde{P}_{\epsilon}^{n, \varphi}$ of the form Eq. (C27) is obtained. The resulting coefficients $\tilde{N}_{\mathrm{s}}^{(k)}$ and $\tilde{N}_{\mathrm{c}}^{(k)}$ for $k \leqslant 5$ are

$$
\begin{aligned}
& \tilde{N}_{\mathrm{s}}^{(0)}(\tilde{x})=\frac{\pi}{4}, \quad \tilde{N}_{\mathrm{c}}^{(0)}(\tilde{x})=0 \\
& \tilde{N}_{\mathrm{s}}^{(1)}(\tilde{x})=-\frac{\pi}{8} \tilde{x} \tilde{E}_{1}, \quad \tilde{N}_{\mathrm{c}}^{(1)}(\tilde{x})=0, \\
& \tilde{N}_{\mathrm{s}}^{(2)}(\tilde{x})=\frac{1}{32 \pi}\left\{\left[\pi^{2}\left(\tilde{x}^{2}-1\right)+8\right] \tilde{E}_{1}^{2}+\left[-4 \pi^{2}\left(\tilde{x}^{2}-1\right)-32\right] \tilde{E}_{2}\right\}, \\
& \tilde{N}_{\mathrm{c}}^{(2)}(\tilde{x})=0 \text {, } \\
& \tilde{N}_{\mathrm{s}}^{(3)}(\tilde{x})=\frac{\tilde{x}}{192 \pi}\left\{\left[\pi^{2}\left(-\tilde{x}^{2}+3\right)-24\right] \tilde{E}_{1}^{3}+12\left[\pi^{2}\left(\tilde{x}^{2}-1\right)+12\right] \tilde{E}_{1} \tilde{E}_{2}-24\left(\pi^{2} \tilde{x}^{2}+6\right) \tilde{E}_{3}-24 \dot{\tilde{E}}_{1}\right\}, \\
& \tilde{N}_{\mathrm{c}}^{(3)}(\tilde{x})=\frac{1}{16}\left(\tilde{x}^{2}-1\right)\left(-2 \tilde{E}_{1} \tilde{E}_{2}+6 \tilde{E}_{3}+\dot{\tilde{E}}_{1}\right), \\
& \tilde{N}_{\mathrm{s}}^{(4)}(\tilde{x})=\frac{1}{1536 \pi^{3}}\left\{\left[\pi^{4}\left(\tilde{x}^{4}-6 \tilde{x}^{2}+5\right)+48 \pi^{2}\left(\tilde{x}^{2}-1\right)\right] \tilde{E}_{1}^{4}+24\left[-\pi^{4}\left(\tilde{x}^{2}-1\right)^{2}-8 \pi^{2}\left(3 \tilde{x}^{2}-1\right)\right] \tilde{E}_{1}^{2} \tilde{E}_{2}+96\left[\pi^{4}\left(\tilde{x}^{4}-1\right)\right.\right. \\
& \left.+4 \pi^{2}\left(3 \tilde{x}^{2}+4\right)-48\right] \tilde{E}_{1} \tilde{E}_{3}+16\left[3 \pi^{4}\left(\tilde{x}^{2}-1\right)^{2}+8 \pi^{2}\left(9 \tilde{x}^{2}-1\right)-192\right] \tilde{E}_{2}^{2}+192\left[\pi^{4}\left(-\tilde{x}^{4}+1\right)\right. \\
& \left.\left.\left.-4 \pi^{2}\left(3 \tilde{x}^{2}+5\right)+96\right)\right] \tilde{E}_{4}+96\left[\pi^{2}\left(\tilde{x}^{2}-3\right)+32\right] \tilde{E}_{1} \dot{\tilde{E}}_{1}+64\left[\pi^{2}\left(-3 \tilde{x}^{2}+7\right)-72\right] \dot{\tilde{E}}_{2}\right\}, \\
& \tilde{N}_{\mathrm{c}}^{(4)}(\tilde{x})=\frac{\tilde{x}}{96}\left(-\tilde{x}^{2}+1\right)\left[-6 \tilde{E}_{1}^{2} \tilde{E}_{2}+30 \tilde{E}_{1} \tilde{E}_{3}+8 \tilde{E}_{2}^{2}-48 \tilde{E}_{4}+3 \tilde{E}_{1} \dot{\tilde{E}}_{1}-4 \dot{\tilde{E}}_{2}\right], \\
& \tilde{N}_{\mathrm{s}}^{(5)}(\tilde{x})=\frac{1}{15360 \pi^{3}} \tilde{x}\left\{\tilde{E}_{1}^{5} \pi^{2}\left[-\pi^{2} \tilde{x}^{4}+\tilde{x}^{2} 10\left(\pi^{2}-8\right)-25 \pi^{2}+240\right]+40 \tilde{E}_{1}^{3} \tilde{E}_{2}\left[(\pi \tilde{x})^{4}-4(\pi \tilde{x})^{2}\left(\pi^{2}-11\right)\right.\right. \\
& \left.+3 \pi^{4}-36 \pi^{2}+96\right]+240 \tilde{E}_{1}^{2} \tilde{E}_{3}\left[-(\pi \tilde{x})^{4}+(\pi \tilde{x})^{2}\left(\pi^{2}-26\right)+2 \pi^{4}-26 \pi^{2}+48\right] \\
& +960 \tilde{E}_{1} \tilde{E}_{4}\left[(\pi \tilde{x})^{4}+20(\pi \tilde{x})^{2}-\pi^{4}+20 \pi^{2}-144\right]+80 \tilde{E}_{1} \tilde{E}_{2}^{2} \pi^{2}\left[-3 \pi^{2} \tilde{x}^{4}+6 \tilde{x}^{2}\left(\pi^{2}-16\right)-3 \pi^{2}+32\right] \\
& -240 \tilde{E}_{1}^{2} \dot{\tilde{E}}_{1}\left[(\pi \tilde{x})^{2}-7 \pi^{2}+72\right]+320 \tilde{E}_{1} \dot{\tilde{E}}_{2}\left[(\pi \tilde{x})^{2}-\pi^{2}+96\right] \\
& +320 \dot{\tilde{E}}_{1} \tilde{E}_{2}\left[(\pi \tilde{x})^{2}+3 \pi^{2}+48\right]+960 \tilde{E}_{2} \tilde{E}_{3}\left[(\pi \tilde{x})^{4}+(\pi \tilde{x})^{2}\left(-\pi^{2}+26\right)-6 \pi^{2}-24\right] \\
& \left.-5760 \dot{\tilde{E}}_{3}\left(\pi^{2}+2\right)+1920 \tilde{E}_{5}\left[-(\pi \tilde{x})^{4}-20(\pi \tilde{x})^{2}+120\right]\right\}, \\
& \tilde{N}_{\mathrm{c}}^{(5)}(\tilde{x})=\frac{1}{384 \pi^{2}}\left(\tilde{x}^{2}-1\right)\left\{-6 \tilde{E}_{1}^{3} \tilde{E}_{2}\left[(\pi \tilde{x})^{2}-\pi^{2}+8\right]+6 \tilde{E}_{1}^{2} \tilde{E}_{3}\left[7(\pi \tilde{x})^{2}-3 \pi^{2}+24\right]\right. \\
& -48 \tilde{E}_{1} \tilde{E}_{4}\left[3(\pi \tilde{x})^{2}+\pi^{2}-12\right]+8 \tilde{E}_{1} \tilde{E}_{2}^{2}\left[5(\pi \tilde{x})^{2}-3 \pi^{2}+24\right]+3 \tilde{E}_{1}^{2} \dot{\tilde{E}}_{1}\left[(\pi \tilde{x})^{2}-\pi^{2}+8\right]-8 \tilde{E}_{1} \dot{\tilde{E}}_{2}\left[(\pi \tilde{x})^{2}+12\right] \\
& \left.-12 \dot{\tilde{E}}_{1} \tilde{E}_{2}\left[(\pi \tilde{x})^{2}-\pi^{2}+16\right]+144 \tilde{E}_{2} \tilde{E}_{3}\left[-(\pi \tilde{x})^{2}+2\right]+12 \dot{\tilde{E}}_{3}\left[(\pi \tilde{x})^{2}+\pi^{2}+12\right]+240 \tilde{E}_{5}\left[(\pi \tilde{x})^{2}+\pi^{2}-12\right]\right\},
\end{aligned}
$$



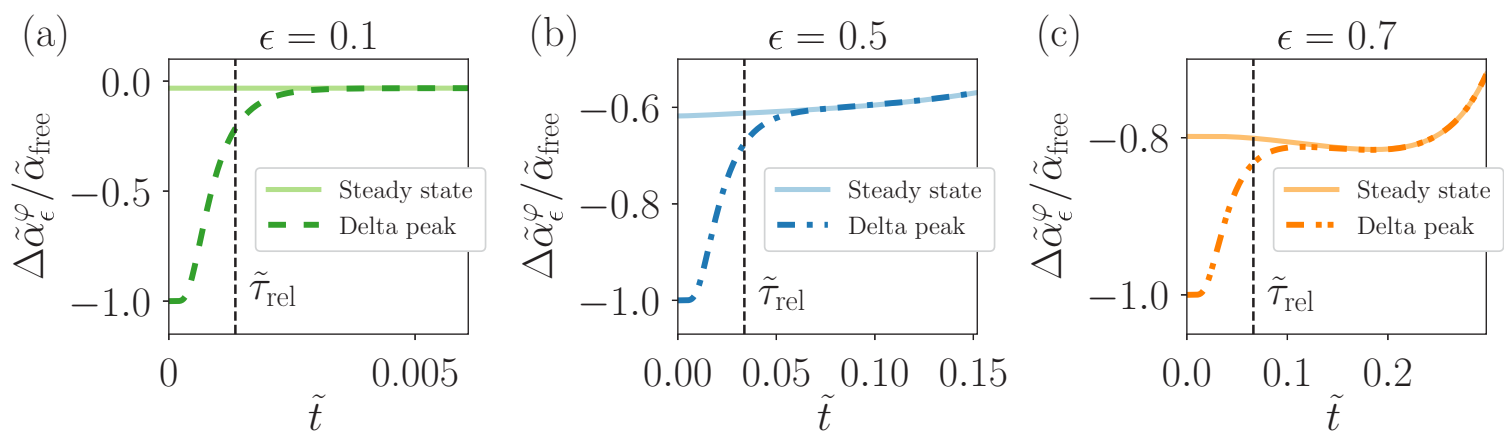

FIG. 5. Effect of the initial distribution $\tilde{P}_{i}$ inside the tube on the exit rate. The exit rate $\tilde{\alpha}_{\epsilon}^{\varphi}$, defined in Eq. (41), is shown as a function of time $\tilde{t}$, for tube radius (a) $\epsilon=0.1$, (b) $\epsilon=0.5$, and (c) $\epsilon=0.7$. From all rates the free-diffusion exit rate is subtracted and the result is divided by the free-diffusion exit rate, as defined in Eq. (57). All data shown are obtained from numerical simulations of the FPE, Eq. (19), from which the exit rate is calculated using Eq. (41). Colored solid lines are replots of the corresponding curves in Figs. 3(d)-3(f), and denote exit rates obtained using the instantaneous steady state as initial condition for the simulations, as explained in Appendix C 5. Colored dashed lines show exit rates obtained using a delta peak at the tube center as initial condition for the simulations. Vertical dashed lines denote the initial relaxation time $\tilde{\tau}_{\text {rel }}$ given in Eq. (C38).

where the $\tilde{E}_{l} \equiv \tilde{E}_{l}(\tilde{t})$ are defined in Eq. (C3) and a dot denotes a derivative with respect to $\tilde{t}$.

\section{Effect of initial distribution inside the tube on exit rate}

As described in Appendix C 5, in the numerical examples in the main text we eliminate transient relaxation effects at the initial time $\tilde{t}_{i}$ by using the instantaneous FP steady state at $\tilde{t}_{i}$ as initial distribution $\tilde{P}_{i}$.

To illustrate the effect of the initial distribution $\tilde{P}_{i}$ on the finite-radius exit rate $\tilde{\alpha}_{\epsilon}^{\varphi}$ we here numerically consider the initial condition $\tilde{P}_{i}(\tilde{x})=\delta(\tilde{x})$, which corresponds to a particle starting out at time $\tilde{t}_{i}$ at the center of the tube.

In Fig. 5 we compare numerical exit rates resulting from this delta-peak initial condition (dashed colored lines) to numerical exit rate corresponding to the instantaneous steadystate initial condition (solid colored lines). As in Figs. 3(d)3(f), we shift and rescale exit rates according to Eq. (57). Using the perturbative results from Appendix $\mathrm{C} 1$, the initial relaxation time $\tilde{\tau}_{\text {rel }}$, defined in Eq. (B47), is given as power series in $\epsilon$ as

$$
\tilde{\tau}_{\text {rel }}=\frac{3 \epsilon^{2}}{4 \pi^{2}}+\mathcal{O}\left(\epsilon^{5}\right) .
$$

This perturbative expression for $\tilde{\tau}_{\text {rel }}$ is plotted in Fig. 5 as vertical dashed lines. Figure 5(a) shows data for tube radius $\epsilon=0.1$. While the data corresponding to the steady-state initial condition (colored solid line) are practically constant on the time scale depicted, the exit rate corresponding to the delta-peak initial condition (colored dashed line) shows relaxation behavior; the curve starts at $\epsilon^{2} \Delta \tilde{\alpha}_{\epsilon}^{\varphi} / \tilde{\alpha}_{\text {free }}(0)=-1$, which according to Eq. (57) corresponds to a vanishing exit rate $\tilde{\alpha}_{\epsilon}^{\varphi}(0)=0$, consistent with the intuition that a particle starting in the center of a finite-radius ball needs a finite time to diffuse out of the ball. This exit rate then relaxes to the steady-state exit rate on a time scale well approximated by Eq. (C38); for times larger than $\tilde{t} \approx 2 \tilde{\tau}_{\text {rel }}$ all knowledge of the initial condition has decayed and the two exit rates are indistinguishable. The data shown for the larger tube radii $\epsilon=0.5$ and 0.7 in Figs. 5(b) and 5(c) show the exact same behavior. As expected from the leading-order scaling $\tilde{\tau}_{\text {rel }} \sim$ $\epsilon^{2}$, the relaxation time increases with tube radius $\epsilon$.

\section{Numerical algorithm for the one-dimensional FPE}

To simulate the dimensionless FPE, Eqs. (19) and (21), we discretize space by introducing the grid

$$
\tilde{x}_{i} \equiv-1+i \Delta \tilde{x} \equiv-1+i \frac{2}{N+1}, \quad i \in\{0, \ldots, N+1\},
$$

and discretize time using a time step $\Delta \tilde{t}$ :

$$
\tilde{t}_{j} \equiv j \Delta \tilde{t}, \quad j \in\left\{\left\lfloor\frac{\tilde{t}_{i}}{\Delta \tilde{t}}\right\rfloor,\left\lfloor\frac{\tilde{t}_{i}}{\Delta \tilde{t}}\right\rfloor+1, \ldots,\left\lfloor\frac{\tilde{t}_{f}}{\Delta \tilde{t}}\right\rfloor\right\},
$$

where \lfloor\rfloor means we round down to the closest integer. Introducing the discretized probability $\tilde{P}_{\epsilon, i}^{j} \equiv \tilde{P}_{\epsilon}^{\varphi}\left(\tilde{x}_{i}, \tilde{t}_{j}\right)$, where $i=1, \ldots, N$, we discretize the FPE, Eq. (19), as

$$
\begin{aligned}
\epsilon^{2} \frac{\tilde{P}_{\epsilon, i}^{j+1}-\tilde{P}_{\epsilon, i}^{j}}{\Delta \tilde{t}}= & \frac{\tilde{P}_{\epsilon, i+1}^{j}-2 \tilde{P}_{\epsilon, i}^{j}+\tilde{P}_{\epsilon, i-1}^{j}}{\Delta \tilde{x}^{2}} \\
& -\epsilon \frac{\tilde{F}_{\mathrm{app}, i+1}^{j} \tilde{P}_{\epsilon, i+1}^{j}-\tilde{F}_{\mathrm{app}, i-1}^{j} \tilde{P}_{\epsilon, i-1}^{j}}{2 \Delta \tilde{x}},
\end{aligned}
$$

where $i \in\{1, \ldots, N\}, \tilde{F}_{\text {app }, i}^{j} \equiv \tilde{F}_{\text {app }}\left(\tilde{x}_{i}, \tilde{t}_{j}\right)$, and in accordance with the absorbing boundary conditions we define $\tilde{P}_{\epsilon, 0}^{j}=$ $\tilde{P}_{\epsilon, N+1}^{j}=0$ for all $j$. To obtain an explicit formula for the distribution at time $(j+1) \Delta \tilde{t}$ in terms of the distribution at time $j \Delta \tilde{t}$, Eq. (C41) is then solved for $\tilde{P}_{\epsilon, i}^{j+1}$ (forward Euler integration scheme).

All numerical results in this paper are obtained using $N=$ 100, $\Delta \tilde{t}=10^{-7}$.

To eliminate boundary effects due to the transient decay of the initial condition, we preequilibrate the system for every $\epsilon$. Starting from a distribution $\tilde{P}_{i}(\tilde{x})=\sin (\pi(\tilde{x}+1) / 2)$, we simulate the FPE, Eq. (C41), for a short time of the order of $\tau_{\text {rel }}$, while holding the parameters for position and velocity of the path $\tilde{\varphi}$ constant at the initial values $\tilde{\varphi}\left(\tilde{t}_{i}\right)$ and $\dot{\tilde{\varphi}}\left(\tilde{t}_{i}\right)$. At the end of this preequilibration, the system is in the instantaneous steady-state decay corresponding to $\tilde{\varphi}\left(\tilde{t}_{i}\right)$ and 
$\dot{\tilde{\varphi}}\left(\tilde{t}_{i}\right)$. This instantaneous steady state is then normalized and used as initial condition for the simulation (in which $\tilde{\varphi}$ and $\dot{\tilde{\varphi}}$ then vary with time). A brief discussion on the dependence of the exit rate on the initial condition is given in Appendix C4.
[1] H. Risken, The Fokker-Planck Equation: Methods of Solution and Applications (Springer, New York, 1996).

[2] B. K. Øksendal, Stochastic Differential Equations: An Introduction with Applications, 6th ed. (Springer, New York, 2007).

[3] N. G. van Kampen, Stochastic Processes in Physics and Chemistry, 3rd ed., North-Holland Personal Library (Elsevier, Amsterdam, 2007).

[4] C. W. Gardiner, Stochastic Methods: A Handbook for the Natural and Social Sciences, 4th ed., Springer Series in Synergetics (Springer, New York, 2009).

[5] J. B. Perrin, Mouvement Brownien et réalité moléculaire, Ann. Chim. Phys. 18, 5 (1909).

[6] M. D. Haw, Colloidal suspensions, Brownian motion, molecular reality: A short history, J. Phys.: Condens. Matter 14, 7769 (2002).

[7] P. Nelson, M. Radosavljević, S. Bromberg, and D. S. Goodsell, Biological Physics: Energy, Information, Life (Freeman, New York, 2014).

[8] S. Bera, S. Paul, R. Singh, D. Ghosh, A. Kundu, A. Banerjee, and R. Adhikari, Fast Bayesian inference of optical trap stiffness and particle diffusion, Sci. Rep. 7, 41638 (2017).

[9] A. Caciagli, D. Joshi, J. Kotar, and E. Eiser, Optical trapping of colloids at a liquid-liquid interface, arXiv:1703.08210.

[10] J. C. M. Gebhardt, T. Bornschlogl, and M. Rief, Full distanceresolved folding energy landscape of one single protein molecule, Proc. Natl. Acad. Sci. USA 107, 2013 (2010).

[11] I. König, A. Zarrine-Afsar, M. Aznauryan, A. Soranno, B. Wunderlich, F. Dingfelder, J. C Stüber, A. Plückthun, D. Nettels, and B. Schuler, Single-molecule spectroscopy of protein conformational dynamics in live eukaryotic cells, Nat. Methods 12, 773 (2015).

[12] A. Amitai and D. Holcman, Polymer physics of nuclear organization and function, Phys. Rep. 678, 1 (2017).

[13] J. Kappler, F. Noé, and R. R. Netz, Cyclization and Relaxation Dynamics of Finite-Length Collapsed Self-Avoiding Polymers, Phys. Rev. Lett. 122, 067801 (2019).

[14] I. S. Aranson, Active colloids, Phys. Usp. 56, 79 (2013).

[15] D. B. Brückner, A. Fink, C. Schreiber, P. J. F. Röttgermann, J. O. Rädler, and C. P. Broedersz, Stochastic nonlinear dynamics of confined cell migration in two-state systems, Nat. Phys. 15, 595 (2019).

[16] Large Deviations and Asymptotic Methods in Finance, Springer Proceedings in Mathematics and Statistics Vol. 110, edited by P. K. Friz, J. Gatheral, A. Gulisashvili, A. Jacquier, and J. Teichmann (Springer, New York, 2015).

[17] B. C. Nolting and K. C. Abbott, Balls, cups, and quasipotentials: Quantifying stability in stochastic systems, Ecology 97, 850 (2016).

[18] U. Seifert, Entropy Production Along a Stochastic Trajectory and an Integral Fluctuation Theorem, Phys. Rev. Lett. 95, 040602 (2005).

[19] U. Seifert, Stochastic thermodynamics, fluctuation theorems, and molecular machines, Rep. Prog. Phys. 75, 126001 (2012).
[20] Weinan E, W. Ren, and E. Vanden-Eijnden, String method for the study of rare events, Phys. Rev. B 66, 052301 (2002).

[21] W. Ren, E. Vanden-Eijnden, P. Maragakis, and Weinan E, Transition pathways in complex systems: Application of the finite-temperature string method to the alanine dipeptide, J. Chem. Phys. 123, 134109 (2005).

[22] Weinan E, W. Ren, and E. Vanden-Eijnden, Transition pathways in complex systems: Reaction coordinates, isocommittor surfaces, and transition tubes, Chem. Phys. Lett. 413, 242 (2005).

[23] L. Onsager and S. Machlup, Fluctuations and irreversible processes, Phys. Rev. 91, 1505 (1953).

[24] R. Graham, Path integral formulation of general diffusion processes, Z. Phys. B 26, 281 (1977).

[25] F. Langouche, D. Roekaerts, and E. Tirapegui, Functional integral methods for stochastic fields, Physica A 95, 252 (1979).

[26] H. Dekker, On the path integral for diffusion in curved spaces, Physica A 103, 586 (1980).

[27] M. F. Weber and E. Frey, Master equations and the theory of stochastic path integrals, Rep. Prog. Phys. 80, 046601 (2017).

[28] L. F. Cugliandolo, V. Lecomte, and F. Van Wijland, Building a path-integral calculus: A covariant discretization approach, J. Phys. A: Math. Theor. 52, 50LT01 (2019).

[29] R. L. Stratonovich, On the probability functional of diffusion processes, Selected Trans. Math. Stat. Prob. 10, 273 (1971).

[30] W. Horsthemke and A. Bach, Onsager-Machlup function for one dimensional nonlinear diffusion processes, Z. Phys. B 22, 189 (1975).

[31] D. Dürr and A. Bach, The Onsager-Machlup function as Lagrangian for the most probable path of a diffusion process, Commun. Math. Phys. 60, 153 (1978).

[32] H. Ito, Probabilistic construction of Lagrangean of diffusion process and its application, Prog. Theor. Phys. 59, 725 (1978).

[33] Y. Takahashi and S. Watanabe, The probability functionals (Onsager-Machlup functions) of diffusion processes, in Stochastic Integrals, edited by D. Williams, Lecture Notes in Mathematics Vol. 851 (Springer, Berlin, Heidelberg, 1981), pp. 433-463.

[34] T. Fujita and S.-I. Kotani, The Onsager-Machlup function for diffusion processes, J. Math. Kyoto University 22, 115 (1982).

[35] N. Ikeda and S. Watanabe, Stochastic Differential Equations and Diffusion Processes, 2nd ed., North-Holland Mathematical Library Vol. 24 (North-Holland, Amsterdam, 1989).

[36] L. Tisza and I. Manning, Fluctuations and irreversible thermodynamics, Phys. Rev. 105, 1695 (1957).

[37] A. W. C. Lau and T. C. Lubensky, State-dependent diffusion: Thermodynamic consistency and its path integral formulation, Phys. Rev. E 76, 011123 (2007).

[38] C. Wissel, Manifolds of equivalent path integral solutions of the Fokker-Planck equation, Z. Phys. B 35, 185 (1979).

[39] Artur B. Adib, Stochastic actions for diffusive dynamics: Reweighting, sampling, and minimization, J. Phys. Chem. B 112, 5910 (2008).

[40] O. Zeitouni, On the Onsager-Machlup functional of diffusion processes around non $C^{2}$ curves, Ann. Probab. 17, 1037 (1989). 
[41] F. Bouchet, K. Gawedzki, and C. Nardini, Perturbative calculation of quasi-potential in non-equilibrium diffusions: A meanfield example, J. Stat. Phys. 163, 1157 (2016).

[42] L. E. Ballentine, Quantum Mechanics: A Modern Development, Reprint 2nd ed. (World Scientific, Singapore, 2010).

[43] See Supplemental Material at http://link.aps.org/supplemental/ 10.1103/PhysRevResearch.2.023407 for videos for the 1D system studied in the present paper.

[44] A. D. Ventsel' and M. I. Freidlin, On small random perturbations of dynamical systems, Russ. Math. Surv. 25, 1 (1970).

[45] T. Li, S. Kheifets, D. Medellin, and M. G. Raizen, Measurement of the instantaneous velocity of a Brownian particle, Science 328, 1673 (2010).
[46] J. Gladrow, M. Ribezzi-Crivellari, F. Ritort, and U. F. Keyser, Experimental evidence of symmetry breaking of transition-path times, Nat. Commun. 10, 55 (2019).

[47] L. Dabelow, S. Bo, and R. Eichhorn, Irreversibility in Active Matter Systems: Fluctuation Theorem and Mutual Information, Phys. Rev. X 9, 021009 (2019).

[48] J. Cresson, Non-differentiable deformations of $\mathbb{P}^{n}$, Int. J. Geometric Meth. Mod. Phys. 03, 1395 (2006).

[49] J. J. Sakurai and J. Napolitano, Modern Quantum Mechanics, 2nd ed. (Cambridge University, Cambridge, England, 2017).

[50] D. S. Grebenkov and B.-T. Nguyen, Geometrical structure of Laplacian eigenfunctions, SIAM Rev. 55, 601 (2013). 